\title{
T2R38 taste receptor polymorphisms underlie susceptibility to upper respiratory infection
}

Robert J. Lee, ${ }^{1}$ Guoxiang Xiong, ${ }^{2}$ Jennifer M. Kofonow, ${ }^{1}$ Bei Chen, ${ }^{1}$ Anna Lysenko, ${ }^{3}$ Peihua Jiang, ${ }^{3}$ Valsamma Abraham, ${ }^{4}$ Laurel Doghramji, ${ }^{1}$ Nithin D. Adappa, ${ }^{1}$ James N. Palmer, ${ }^{1}$ David W. Kennedy, Gary K. Beauchamp, ${ }^{3}$ Paschalis-Thomas Doulias, ${ }^{4,5}$ Harry Ischiropoulos, ${ }^{4,5}$ James L. Kreindler, ${ }^{4}$ Danielle R. Reed, ${ }^{3}$ and Noam A. Cohen ${ }^{1,6}$

1Department of Otorhinolaryngology, Head and Neck Surgery, University of Pennsylvania, Philadelphia, Pennsylvania, USA. 2Division of Neurology, Children's Hospital of Philadelphia, Pennsylvania, USA. ${ }^{3}$ Monell Chemical Senses Center, Philadelphia, Pennsylvania, USA. ${ }^{4}$ Department of Pediatrics, Children's Hospital of Philadelphia, Pennsylvania, USA. ${ }^{5}$ Department of Pharmacology, University of Pennsylvania, Philadelphia, Pennsylvania, USA. ${ }^{6}$ Philadelphia Veterans Affairs Medical Center, Surgical Services, Philadelphia, Pennsylvania, USA.

\begin{abstract}
Innate and adaptive defense mechanisms protect the respiratory system from attack by microbes. Here, we present evidence that the bitter taste receptor T2R38 regulates the mucosal innate defense of the human upper airway. Utilizing immunofluorescent and live cell imaging techniques in polarized primary human sinonasal cells, we demonstrate that T2R38 is expressed in human upper respiratory epithelium and is activated in response to acyl-homoserine lactone quorum-sensing molecules secreted by Pseudomonas aeruginosa and other gram-negative bacteria. Receptor activation regulates calcium-dependent NO production, resulting in stimulation of mucociliary clearance and direct antibacterial effects. Moreover, common polymorphisms of the TAS2R38 gene were linked to significant differences in the ability of upper respiratory cells to clear and kill bacteria. Lastly, TAS2R38 genotype correlated with human sinonasal gram-negative bacterial infection. These data suggest that T2R38 is an upper airway sentinel in innate defense and that genetic variation contributes to individual differences in susceptibility to respiratory infection.
\end{abstract}

\section{Introduction}

Inhaled pathogens, toxins, and particulates continuously challenge the respiratory system. The principal physical defense against these inhaled insults is mucociliary clearance (MCC), which has 2 components, mucus production and mucus transport $(1,2)$. Coordinated ciliary beating transports debris-laden mucus from both the upper and lower respiratory passages toward the oropharynx, from which it is cleared by expectoration or swallowing (3). Ciliary beating accelerates in response to multiple host and environmental stimuli (4-14) through several second messenger pathways including intracellular $\mathrm{Ca}^{2+}(15-17)$ and $\mathrm{NO}$ production $(8,18,19)$. In addition to its role as a second messenger, NO diffuses into the airways where it has antimicrobial properties and thus is central to host defense against respiratory infections (20).

In response to multiple environmental factors, microbes secrete chemical signals, known as autoinducers or quorum-sensing molecules, which regulate the expression of microbial genes involved in biofilm formation, persistence, virulence, and other life cycle processes $(21,22)$. Given the importance of these molecules in pathogenicity, there is strong biological plausibility that such molecules are sensed and responded to by the host.

The pathogen-derived triggers of respiratory host defenses are likely to include those that humans report to taste bitter, based on recent observations that bitter taste receptors (T2Rs) are expressed in both upper (23) and lower human respiratory epithelium (24). Additionally, in vitro stimulation of human lower airway cultures with various bitter-tasting compounds increased intracellular $\mathrm{Ca}^{2+}$ and ciliary beat frequency (CBF) (24). To date, natural ligands of T2Rs in the human airway have not been identified. We hypoth-

Conflict of interest: The authors have declared that no conflict of interest exists. Citation for this article: J Clin Invest. 2012;122(11):4145-4159. doi:10.1172/JCI64240. esize that possible ligands for human airway T2Rs include chemicals secreted by microbes, such as quorum-sensing molecules, a hypothesis supported by previous work in mice $(25,26)$.

Preliminary screening studies identified prominent expression of T2R38, the receptor for the compound phenylthiocarbamide (PTC), in upper airway epithelium. Bitter receptors, and particularly T2R38, are unique among G protein-coupled receptors in the density of their naturally occurring genetic variants (27). Many previous studies have demonstrated that people with the taster form of this receptor report that concentrations of some bitter ligands like PTC are intensely bitter at concentrations that are imperceptible to those with the non-taster form (28). The differences between the taster and non-taster form lie in the amino acid residues at positions 49, 262, and 296; the functional allele of the receptor contains a proline, alanine, and valine (PAV), while the nonfunctional allele of the receptor contains an alanine, valine, and isoleucine (AVI) at these positions, respectively (29). Based on bioinformatics and modeling, it has been suggested that the valine at the third position in the polymorphism is involved in interhelical hydrogen bonding that stabilizes the receptor structure and allows receptor activation only in tasters $(30,31)$, despite a likely similar binding affinity of PTC for both forms of the receptor (32). Thus, we further hypothesized that functional differences in taste perception predict functional responses in the airway to pathogen-derived quorum-sensing molecules. If true, genetically determined individual taste perception could identify individuals susceptible to bacterial respiratory infections and may guide alternative therapeutic interventions.

While mice have bitter receptors that are capable of responding to PTC to some extent (33), they do not have a clear T2R38 homolog, and the mechanisms underlying their avoidance of PTC are not as well defined $(34,35)$, thus eliminating potential stud- 
A

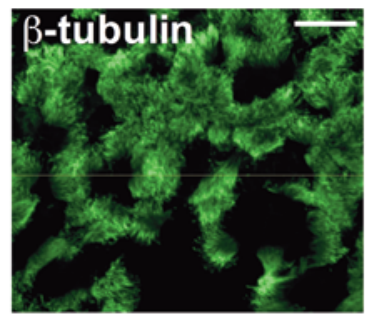

B

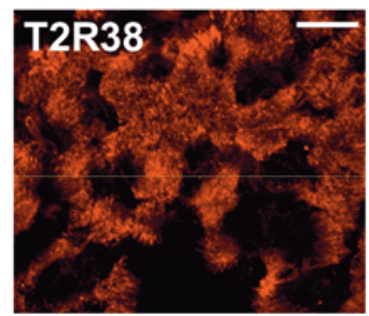

C

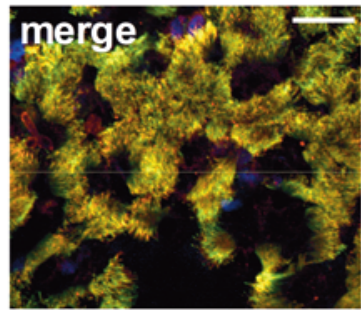

D
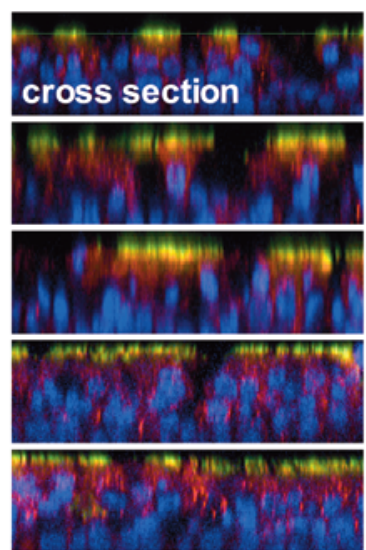

I

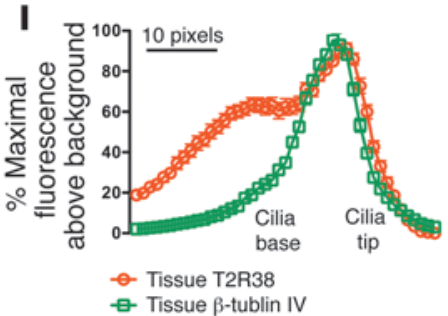

— Tissue $\beta$-tublin IV
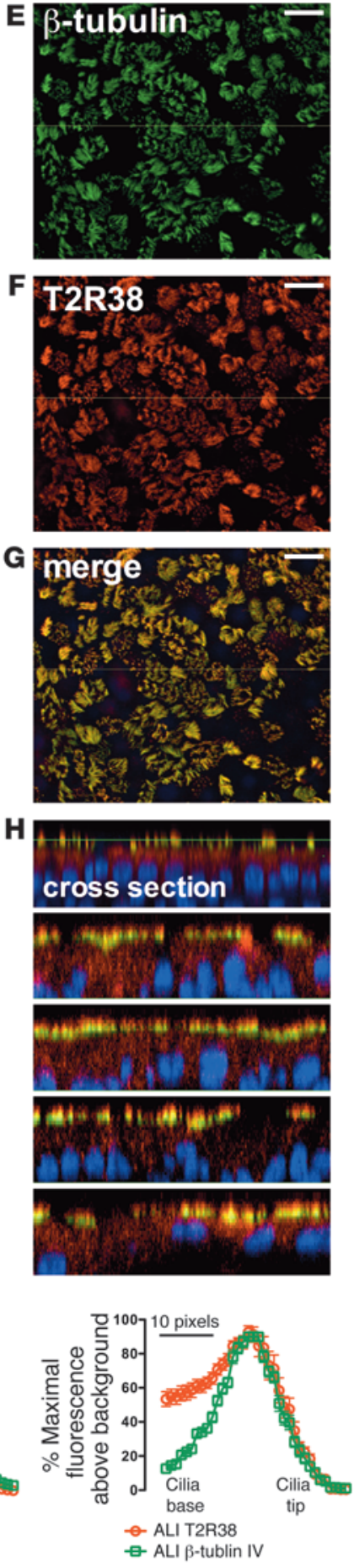

ies in knockout animals and necessitating use of a human model system. To test our hypotheses, we used primary human sinonasal cells genotyped for TAS2R38 (encoding T2R38) and cultured at an air-liquid interface (ALI), recapitulating the polarized respiratory epithelium. ALI cultures are a state-of-the-art epithelial model that has been used for extensive studies of the upper (36) and lower airways (37). We measured three indexes of respiratory innate defense, $\mathrm{NO}$ production, mucociliary clearance, and bactericidal activity. We found that T2R38 plays a critical role in the

\section{Figure 1}

T2R38 is expressed at the apical membrane and cilia of sinonasal airway epithelial cells in both human tissue explants and primary human sinonasal ALI cultures. (A-H) Representative images of $\beta$-tubulin (green; a ciliary marker), T2R38 (red), and Hoechst (blue; a nuclear stain) in primary human sinonasal tissue (A-D) and in a human sinonasal ALI culture (E-H). Scale bars: $20 \mu \mathrm{m}$. The height of the crosssection is stretched to illustrate the co-localization pattern. $\mathbf{D}$ and $\mathbf{H}$ show cross-sections of z-projections from 4 other tissue samples (D) and from cultures $(\mathbf{H})$ illustrating the co-localization pattern. (I) 32 regions of ciliated cells were analyzed for red and green fluorescence (left to right: basolateral to apical) over approximately 40 pixels.

detection and response to quorum-sensing molecules produced by gram-negative bacteria, including the important respiratory pathogen Pseudomonas aeruginosa. Differences in T2R38 functionality, as assessed by TAS2R38 genotype, significantly correlated with differential susceptibilities of patients to gram-negative bacterial sinonasal infection.

\section{Results}

T2R38 is expressed and functional in human upper airway cells. T2R38 expression was observed in the apical membrane and cilia of sinonasal respiratory epithelial cells from surgical explants as well as primary sinonasal ALI cultures (Figure 1$)$. The green ( $\beta$-tubulin IV, a cilia marker) and red (T2R38) fluorescence intensities were analyzed along 32 regions of cilia from $z$-axis projections of tissue (Figure 1D; 4 samples analyzed) and cultures (Figure $1 \mathrm{H}$; 8 cultures analyzed). In both tissue and cultures, red fluorescence (T2R38 expression) began at the tip of the cilia and persisted below the base of the cilia, perhaps reflecting T2R38 in the process of trafficking to the plasma membrane. The Manders correlation coefficient (38) for green staining was $0.96 \pm 0.02$ and $0.99 \pm 0.01$ in tissue and ALI cultures, respectively, suggesting that more than $96 \%$ of green pixels (cilia) were positive for red staining (T2R38). Overall, these data suggest that T2R38 is expressed along the entire length of the cilia in both tissue and ALI cultures.

T2Rs are metabotropic $G$ protein-coupled receptors that can signal through intracellular $\mathrm{Ca}^{2+}(39)$. T2R stimulation (but not T2R38) has been shown to activate $\mathrm{Ca}^{2+}$ signaling in human bronchial epithelial cells (24) as well as rodent nasal solitary chemosensory cells (40). We thus tested whether the T2R38-specific agonist PTC (41) elevated $\mathrm{Ca}^{2+}$ in human sinonasal epithelial cells (HSECs), using the fluorescent calcium indicator Fluo-4 (42). Apical (air-side) stimulation with $1 \mathrm{mM}$ PTC (29) induced low-level, sustained intracellular $\mathrm{Ca}^{2+}$ elevations (Figure 2A and Supplemental Figure 1, A and B; supplemental material available online with this article; doi:10.1172/JCI64240DS1) in the majority of cells, demonstrating functionality of T2R38, while basolateral stimulation or vehicle alone had no effect (Supplemental Figure 1, C and D). Notably, the magnitudes of PTC-induced $\mathrm{Ca}^{2+}$ changes were a function of TAS2R38 genotype (Figure 2, A and B). Cultures derived from tasters (PAV/PAV) exhibited the greatest response, while cultures from non-tasters (AVI/AVI) and heterozygous individuals (PAV/AVI) had nearly undetectable responses. In contrast, purinergic receptor-dependent ATP-induced $\mathrm{Ca}^{2+}$ responses did not correlate with TAS2R38 genotype (Figure 2, A and B, and Supplemental Figure 2) and RT-PCR for TAS2R38 from genotypically distinct cultures demonstrated comparable levels of expression (Supplemental Figure 2D). Likewise, immunofluorescence dem- 

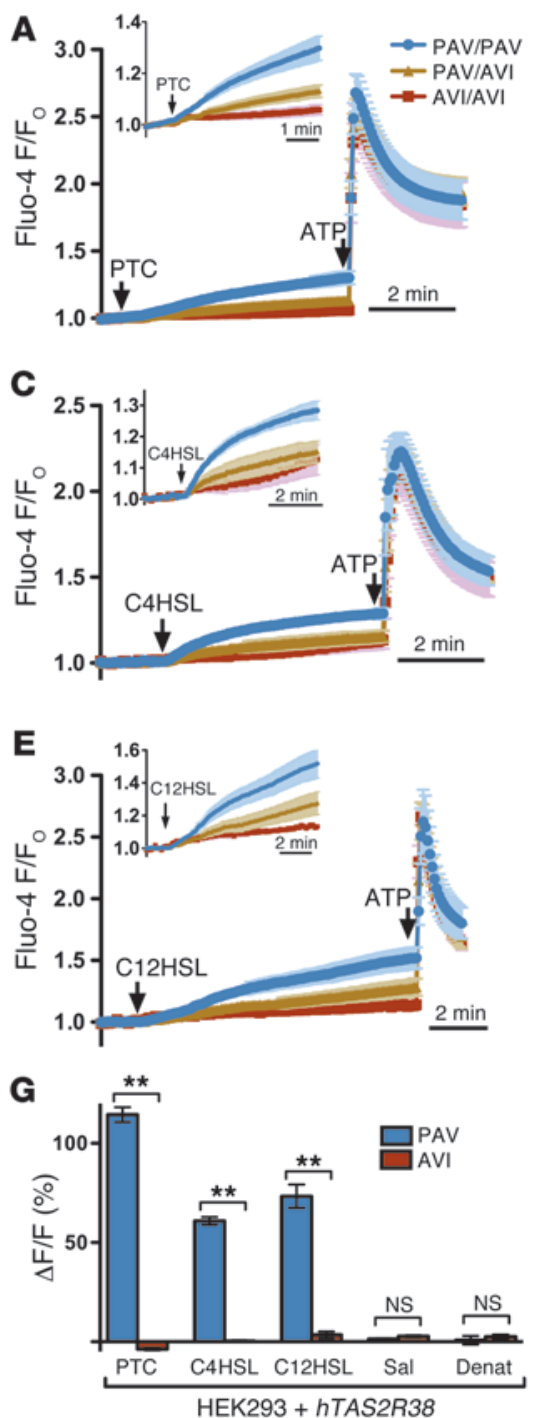

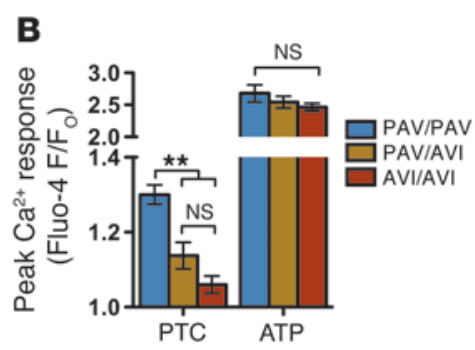

D

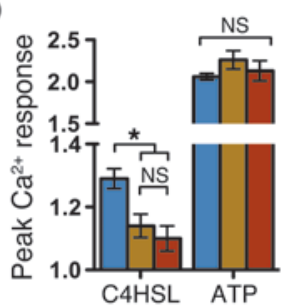

$\mathbf{F}$

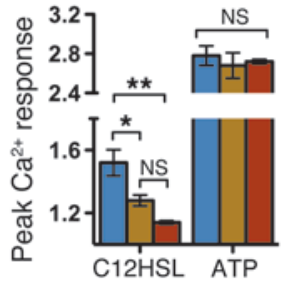

\section{Figure 2}

PTC and Pseudomonas AHLs induce T2R38-dependent $\mathrm{Ca}^{2+}$ responses in sinonasal ALIs and in a heterologous expression system. (A) $\mathrm{Ca}^{2+}$ responses to PTC and ATP stimulation (mean $\pm \mathrm{SEM}$; $12 \mathrm{PAV} / \mathrm{PAV}, 16 \mathrm{PAV} / \mathrm{AVI}$, and $8 \mathrm{AVI} / \mathrm{AVI}$ cultures, $\mathrm{n}=4$ patients each). Inset: PTC responses on a larger scale. (B) Peak $\mathrm{Ca}^{2+}$ responses from patients in $\mathbf{A}$. Results from individual patients were pooled and averaged; each independent observation represents 1 patient. Fluo-4 fluorescence after 5 minutes of PTC: $1.30 \pm 0.027$ (PAV/PAV), $1.14 \pm 0.035$ (PAV/AVI), and $1.06 \pm 0.02$ (AVI/AVI). (C) Fluo-4 traces (means from 10 cultures) during stimulation with $200 \mu \mathrm{M} \mathrm{C} 4 \mathrm{HSL}$ and ATP. (D) Peak $\mathrm{Ca}^{2+}$ responses from $\mathbf{C}$, averaged as described in B. Fluo-4 fluorescence after 5 minutes of C4HSL: $1.29 \pm 0.03(\mathrm{PAV} / \mathrm{PAV}), 1.14 \pm 0.04(\mathrm{PAV} / \mathrm{AVI})$, and $1.10 \pm 0.04(\mathrm{AVI} / \mathrm{AVI})$. ( $E$ and $\mathbf{F})$ Experiments were performed as in C and D using $100 \mu \mathrm{M} \mathrm{C12HSL}$ and 3 cultures from 3 patients/genotype. Fluo-4 fluorescence after 10 minutes of $\mathrm{C} 12 \mathrm{HSL}$ stimulation: $1.52 \pm 0.08$ (PAV/PAV), $1.28 \pm 0.04$ (PAV/AVI), and $1.14 \pm 0.01$ (AVI/AVI). (G) Peak Fluo-4 fluorescence $(\triangle F / F)$ in hTAS2R38- and Ga16gustducin44-expressing HEK293 cells in response to PTC $(114 \% \pm 4 \%$ PAV; $-4 \% \pm 1 \%$ $\mathrm{AVI}), \mathrm{C} 4 \mathrm{HSL}(61 \% \pm 2 \% \mathrm{PAV} ; 0.3 \% \pm 0.4 \% \mathrm{AVI})$, and C12HSL $(73 \% \pm 5 \%$ PAV; $3 \% \pm 2 \%$ AVI), denatonium (Denat; $1 \% \pm 2 \% \mathrm{PAV} ; 3 \% \pm 1 \% \mathrm{AVI}$ ), and sali$\operatorname{cin}(\mathrm{Sal} ; 1.4 \% \pm 0.3 \% \mathrm{PAV} ; 3 \% \pm 1 \% \mathrm{AVI}) .{ }^{*} P<0.05$, ${ }^{* *} P<0.01$, ANOVA with Tukey-Kramer analysis. onstrated comparable T2R38 expression between PAV/PAV and AVI/AVI cultures (Supplemental Figure 2E). This indicates that the reduced PTC-induced $\mathrm{Ca}^{2+}$ responses in heterozygous (PAV/ $\mathrm{AVI}$ ) and homozygous nonfunctional (AVI/AVI) cultures are due to differential activation of the T2R38 protein and not intrinsic defects in $\mathrm{Ca}^{2+}$ signaling or differential expression. T2R38-activated $\mathrm{Ca}^{2+}$ signaling was reduced by inhibition of PLC $\beta 2$, the inositol trisphosphate receptor, or TRPM5, downstream components of receptor signaling in taste cells (refs. 43, 44, and Supplemental Figure 3). These data indicate that T2R38 is expressed in human upper airway epithelium and that homozygosity for the TAS2R38 functional genotype (PAV) is required for maximal T2R38 signal propagation in sinonasal respiratory epithelial cells.

T2R38 detects gram-negative quorum-sensing molecules. Murine nasal chemosensory cells express bitter taste-signaling molecules and detect Pseudomonal quorum-sensing molecules (26). Thus we tested whether these bacterial products activate $\mathrm{Ca}^{2+}$ signaling in HSECs and whether such activation is dependent on the TAS2R38 genotype. Cultures were stimulated with the gram-negative quorum-sensing molecule N-butyryl-L-homoserine lactone (C4HSL) at $200 \mu \mathrm{M}$, or N-3-oxo-dodecanoyl-L-homoserine lac- tone (C12HSL) at $100 \mu \mathrm{M}$, physiological acyl-homoserine lactone (AHL) concentrations observed during late stationary planktonic phase and biofilm growth (45). Both C12HSL and C4HSL are produced by the important respiratory pathogen $P$. aeruginosa as well as other gram-negative bacteria (46-48), which may use these molecules for interspecies communication to form mixed biofilms on the airway epithelium (49). Cultures derived from individuals homozygous for functional T2R38 (PAV/PAV) had a significantly greater $\mathrm{Ca}^{2+}$ response to the AHLs compared with those homozygous or heterozygous for the nonfunctional receptor (AVI/AVI or PAV/AVI, respectively) (Figure 2, C-F). N-hexanoyl-L-homoserine lactone (C6HSL), produced by some $P$. aeruginosa, Burkholderia cenocepacia, and Burkholderia multivorans strains (47, 50, 51), also produced $\mathrm{Ca}^{2+}$ responses that were greater in PAV/PAV cultures than in AVI/AVI cultures that were blocked by PLC $\beta 2$ inhibition (Supplemental Figure 4). Application of 3 concentrations (6.25\%, $12.5 \%, 25 \%$ ) of conditioned medium from a 3 -day biofilm culture of $P$. aeruginosa (strain PAO1) to ALI cultures with functional T2R38 receptors also elicited $\mathrm{Ca}^{2+}$ responses that were blocked by inhibition of PLC $\beta 2$ (Supplemental Figure 5). Sequential addition experiments using high concentrations of PTC $(15 \mathrm{mM})$ and 
A
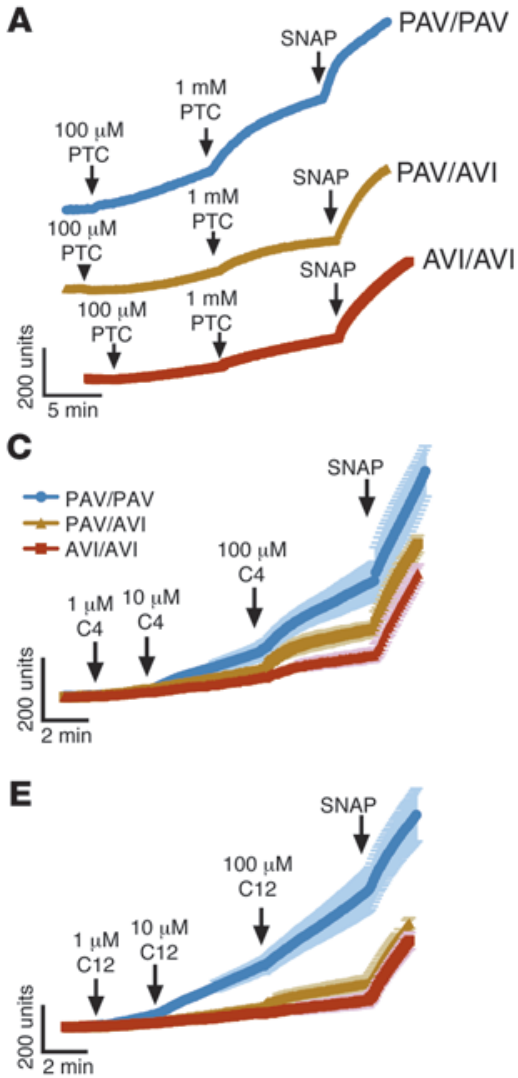

B
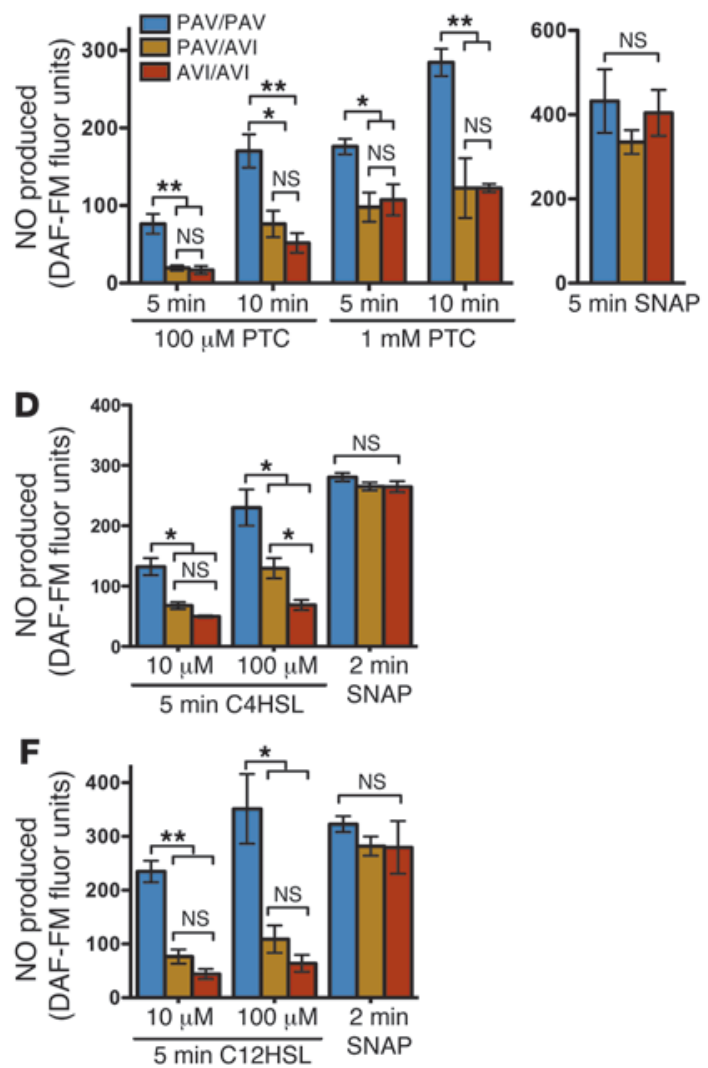

Figure 3

Activation of T2R38 by PTC or Pseudomonas AHLs results in NO production. (A) Traces of DAF-FM fluorescence increased with PTC (1 culture each, 100 cells; SEM smaller than symbols; 9-12 cultures each). (B) DAF-FM fluorescence increased after PTC stimulation. $100 \mu \mathrm{M} / 5$ minutes: $76 \pm 13$ (PAV/PAV), $20 \pm 3$ (PAV/AVI), and $17 \pm 5$ (AVI/AVI); $100 \mu \mathrm{M} / 10$ minutes; $170 \pm 21$ (PAV/PAV), $76 \pm 17$ (PAV/AVI), and $52 \pm 13$ (AVI/AVI); $1 \mathrm{mM} / 5$ minutes: $176 \pm 10$ (PAV/PAV), $98 \pm 19(\mathrm{PAV} / \mathrm{AVI})$, and $107 \pm 20$ (AVI/AVI): $1 \mathrm{mM} / 10$ minutes: $285 \pm 18$ (PAV/PAV), $123 \pm 38$ (PAV/AVI;), and $122 \pm 5$ (AVI/AVI). Increases after SNAP were not different between cultures of different genotypes. Each patient treated as an independent observation; $n=4$ patients each. (C) Average traces of DAF-FM with C4HSL (2 cultures each from 4 patients for each genotype). (D) Results from C averaged by patient. Fluorescence changes after 5 minutes were as follows for $10 \mu \mathrm{M}$ C4HSL: $132 \pm 14$ (PAV/PAV), $68 \pm 6$ (PAV/AVI), $50 \pm 2$ (AVI/AVI); $100 \mu \mathrm{M} \mathrm{C4HSL:}$ $230 \pm 30$ (PAV/PAV), $130 \pm 17$ (PAV/AVI), and $69 \pm 8$ (AVI/AVI). (E) Average traces of DAF-FM with C12HSL (2 cultures each from 4 PAV/PAV patients and 3 PAV/AVI and AVI/AVI patients). (F) Results from $E$ averaged as described in $\mathbf{D}$. Fluorescence changes after 5 minutes were as follows for $10 \mu \mathrm{M}$ C12HSL: $235 \pm 20$ (PAV/PAV), $77 \pm 13$ (PAV/AVI), and $44 \pm 9$ (AVI/AVI); $100 \mu \mathrm{M}$ C12HSL: $351 \pm 65$ (PAV/PAV), $109 \pm 25(\mathrm{PAV} / \mathrm{AVI})$, and $64 \pm 16(\mathrm{AVI} / \mathrm{AVI})$. Increases after SNAP were not significantly different. ${ }^{*} P<0.05,{ }^{\star *} P<0.01$, ANOVA with Tukey-Kramer analysis.

C4HSL $(400 \mu \mathrm{M})$ suggested that these 2 agonists activate $\mathrm{Ca}^{2+}$ signaling through a common pathway (Supplemental Figure 6A). In addition, siRNA knockdown of TAS2R38 expression significantly reduced the C4HSL- and C12HSL-induced $\mathrm{Ca}^{2+}$ responses observed in PAV/PAV cultures (Supplemental Figure 6, B and C), suggesting this receptor is a critical component of airway signaling in response to AHLs.

The onset of the $\mathrm{Ca}^{2+}$ responses to PTC, C4HSL, and C12HSL occurred within one time point ( 5 seconds) after addition of the compounds $90 \%$ of the time, and within 2 frames ( 10 seconds) $10 \%$ of the time. These fast responses suggest that this $\mathrm{Ca}^{2+}$ elevation is a result of direct receptor activation rather than a secondary response of another compound secreted or released in response to C4HSL/C12HSL.
To definitively test whether T2R38 is directly activated by C4HSL and C12HSL, the PAV and AVI variants of human TAS2R 38 (hTAS2R38) were cloned from $\mathrm{PAV} / \mathrm{PAV}$ and AVI/AVI homozygous individuals and expressed in HEK293 cells co-transfected with a chimeric $G$ protein Ga16gustducin44 to couple receptor activation to $\mathrm{Ca}^{2+}$ mobilization (52). This expression system has previously been used to study T2R38, and it has shown that both forms of the protein are targeted to the plasma membrane of HEK293 cells (29). Fluo-4 fluorescence was monitored during stimulation with $1 \mathrm{mM}$ PTC, 200 $\mu \mathrm{M}$ C4HSL, and $100 \mu \mathrm{M}$ C12HSL. The bitter T2R agonists denatonium $(1 \mathrm{mM})$ and salicin $(10 \mathrm{mM})$ were included as controls as they do not activate T2R38 (52). PTC $(1 \mathrm{mM})$, C4HSL $(200 \mu \mathrm{M})$, and C12HSL $(100 \mu \mathrm{M})$ all activated $\mathrm{Ca}^{2+}$ signals in PAV TAS2R38expressing cells, but no response was observed in AVI TAS2R38expressing cells (Figure 2G), strongly supporting the direct activation of T2R38 signaling by gram-negative bacterial AHLs. No significant $\mathrm{Ca}^{2+}$ response was observed with salicin or denatonium, as expected.

Similar to ALI cultures, $\mathrm{Ca}^{2+}$ responses to PTC and C4HSL occurred in acutely dissociated sinonasal ciliated cells (Supplemental Figure 7), indicating that the responses observed in the ALI cultures recapitulate the in vivo epithelial response. Together, these data suggest that T2R38 contributes to the detection of quorum-sensing molecules from gram-negative bacteria in HSECs, perhaps leading to downstream defense mechanisms that are reduced in patients with at least 1 nonfunctional (AVI) TAS2R38 allele.

$T 2 R 38$ activation triggers $N O$ production. Because the above data indicate that T2R 38 plays a role in the detection of gram-negative bacteria by respiratory epithelial cells, we hypothesized that T2R38-induced $\mathrm{Ca}^{2+}$ responses likely activate one or more known antimicrobial pathways. We found that T2R38 activation does not result in cytokine secretion (inflammation) or direct antimicrobial peptide secretion over the course of 12-14 hours of exposure (Supplemental Figure 8). Additionally, we found no significant difference in cytokine secretion between PAV/PAV and AVI/AVI cultures stimulated with Pseudomonas LPS (a TLR4 agonist; $10 \mu \mathrm{g} / \mathrm{ml}$ ) or the 
A
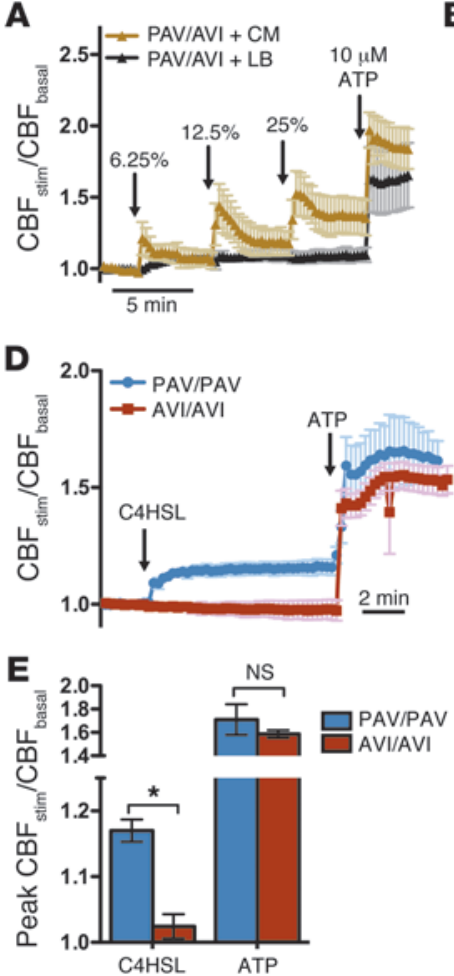

B
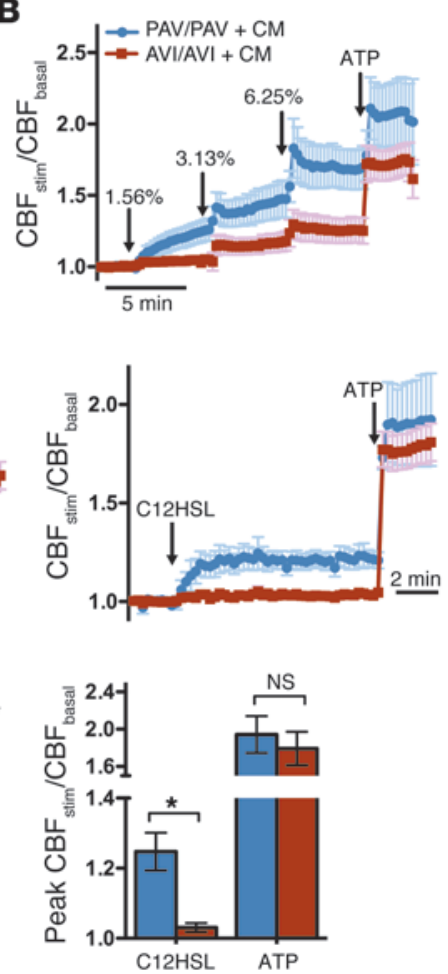

C

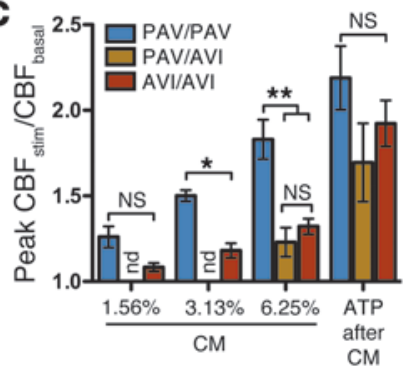

Figure 4

Pseudomonas conditioned medium (CM) and AHLs stimulate an increase in CBF that requires T2R38 function. (A) Average traces showing CBF increase in PAV/AVI cultures stimulated (stim) with CM or LB as well as ATP. (B) Average traces showing the effects of 3 concentrations of CM on $\mathrm{CBF}$ in PAV/PAV taster (9 cultures from 3 patients) and AVI/AVI non-taster cultures (11 cultures from 3 patients). (C) Summary of $\mathbf{A}$ and $\mathbf{B}$, treating each patient as an independent observation ( $n=3$ each; nd, not determined). CBF increased to $1.26 \pm 0.6$ (PAV/PAV) and 1.08 \pm 0.2 (AVI/AVI; $P=$ NS) with $1.56 \% \mathrm{CM} ; 1.50 \pm 0.03$ (PAV/PAV) and $1.18 \pm 0.04(\mathrm{AVI} / \mathrm{AVI} ; P=0.03)$ with $3.13 \% \mathrm{CM}$; and $1.83 \pm 0.11(\mathrm{PAV} / \mathrm{PAV}), 1.23 \pm 0.09(\mathrm{PAV} /$ AVI; $P<0.01$ vs. PAV/PAV), and $1.32 \pm 0.05$ (AVI/AVI; $P<0.01$ vs. PAV/PAV; $P=$ NS vs. PAV/AVI) with $6.25 \%$ CM. Peak CBF after ATP stimulation was not significantly different between cultures of different genotypes. (D) Average traces showing peak CBF in response to $200 \mu \mathrm{M}$ C4HSL and $100 \mu \mathrm{M}$ C12HSL in PAV/PAV and AVI/AVI cultures (6 cultures from 3 patients each). (E) Summary of results from $\mathbf{D}$, treating each patient as an independent observation ( $n=3-4$ patients each). Peak CBF with C4HSL was $1.17 \pm 0.02$ (PAV/PAV) and $1.02 \pm 0.02(\mathrm{AVI} / \mathrm{AVI})$; peak CBF with $\mathrm{C} 12 \mathrm{HSL}$ was $1.25 \pm 0.05$ (PAV/PAV) and $1.03 \pm 0.01$ (AVI/AVI). ${ }^{*} P<0.05,{ }^{\star \star} P<0.01$, ANOVA with Tukey-Kramer analysis.

TLR2/ 6 agonist FSL-1 (1 $\mu \mathrm{g} / \mathrm{ml}$ ) (Supplemental Figure 8), strongly suggesting that T2R38 signaling is independent of TLR signaling and cytokine secretion. Another antimicrobial pathway is NO (20), which can be generated by low-level intracellular $\mathrm{Ca}^{2+}$ changes that stimulate calmodulin-dependent NOS activation (53). We measured cellular NO production using the fluorescent probe 4-amino-5-methylamino-2',7'-difluorescein (DAF-FM), which reacts with $\mathrm{NO}$-derived reactive nitrogen species to form a fluorescent benzotriazole (54). PTC (100 $\mu \mathrm{M}$ and $1 \mathrm{mM})$ stimulation resulted in NO-derived reactive species production (DAF-FM fluorescence) over the course of 10 minutes (Figure 3, A and B). Furthermore, DAF-FM fluorescence increase was a function of T2R38 genotype, with PAV/PAV cultures exhibiting greater responses than either PAV/AVI or AVI/AVI cultures. Exposure to the NO donor S-nitroso-N-acetyl-D,L-penicillamine (SNAP; $100 \mu \mathrm{M}$; Figure 3, $A$ and B) or ionomycin (Supplemental Figure 9) did not yield differential DAF-FM responses between the genotypes, suggesting that differences in PTC-induced DAF-FM fluorescence were not an artifact of differential dye loading or NOS activity. PTC-dependent $\mathrm{NO}$ production was inhibited by blocking PLC $\beta 2$ or downstream $\mathrm{Ca}^{2+}$ signaling (Supplemental Figure 10). Another T2R38 agonist, sodium thiocyanate ( $\mathrm{NaSCN})$, activated similar T2R38-dependent DAF-FM fluorescence that was blocked by the NOS inhibitor $\mathrm{L}_{-} \mathrm{N}^{\mathrm{G}}$ nitroarginine methyl ester (L-NAME) (ref. 55 and Supplemental Figure 11, A and B) despite no effect on T2R38-activated $\mathrm{Ca}^{2+}$ signaling (Supplemental Figure 11, C and D). Two other bitter taste ligands, thujone $(5 \mathrm{mM})$ and denatonium $(10 \mathrm{mM})$, which activate multiple T2Rs but not T2R38 (52), did not stimulate NO production (Supplemental Figure 11, E and F) suggesting that T2R38 functionality is specifically critical for upper respiratory epithelial NO production in response to bitter compounds.

Because sinonasal ALIs stimulated with either Psendomonasconditioned medium or with purified AHLs exhibited T2R38dependent $\mathrm{Ca}^{2+}$ responses, we tested whether AHLs activate T2R38-dependent NO production. C4HSL $(10-100 \mu \mathrm{M})$ produced an increase in DAF-FM fluorescence that was significantly larger in cultures with functional receptors (PAV/PAV) than in cultures with nonfunctional T2R38 (AVI/AVI) (Figure 3, C and D), that was blocked by inhibition of NOS or PLC $\beta 2$ (Supplemental Figure 12). Additionally, siRNAs directed against TAS2R38 expression significantly decreased the NO response to C4HSL (Supplemental Figure 12, E and G). Likewise, T2R38 genotype-dependent NO produc- 

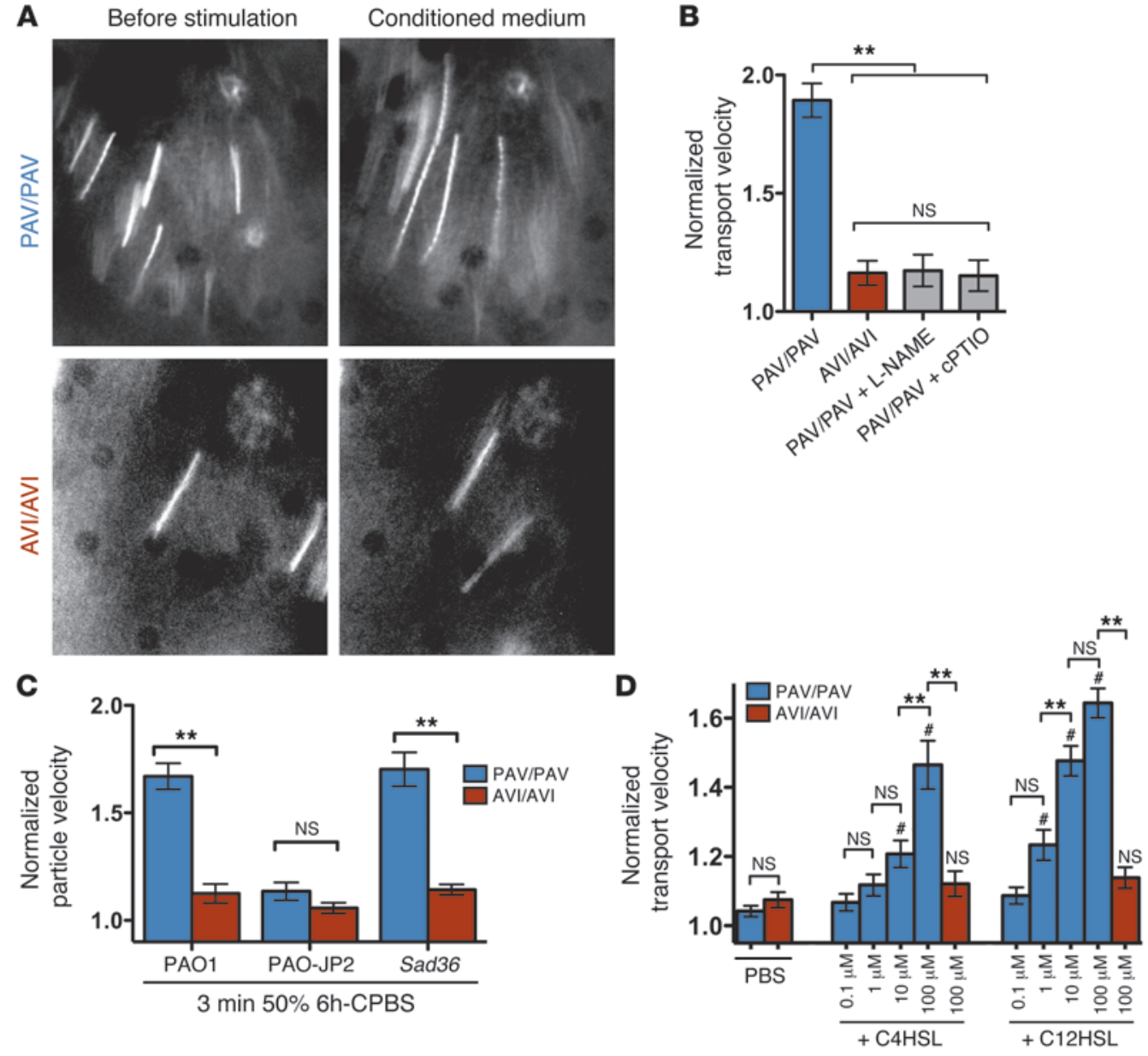

\section{Figure 5}

Pseudomonas CM and AHLs stimulate a T2R38-dependent, NO-dependent increase in mucociliary clearance. (A) Particle streaks before (left) and 3 minutes after (right) stimulation with 6.25\% biofilm-CM in PAV/PAV and AVI/AVI cultures from PAV/PAV and AVI/AVI patients. (B) Mean normalized velocity increase was $1.9 \pm 0.07$ (PAV/PAV), $1.16 \pm 0.05$ (AVI/AVI), $1.17 \pm 0.07$ (PAV/PAV + L-NAME), and $1.15 \pm 0.07$ (PAV/PAV + cPTIO). (C) Results from transport analysis using CPBS (after 3 minute exposure of 50\%, 6 hour CPBS) from PAO1, PAO-JP2, and Sad36 strains (4 cultures/genotype/strain). Normalized velocity increases were as follows for PAO-1: $1.67 \pm 0.06$ (PAV/PAV) vs. 1.13 \pm 0.04 (AVI/AVI); PAO-JP2: $1.135 \pm 0.04$ (PAV/PAV) vs. $1.058 \pm 0.03$ (AVI/AVI); Sad36: $1.703 \pm 0.08$ (PAV/PAV) vs. $1.14 \pm 0.03$ (AVI/AVI). (D) Transport analysis using C4HSL and C12HSL. Increases in velocity upon PBS addition: $1.04 \pm 0.02$ (PAV/PAV) vs. $1.08 \pm 0.2$ (AVI/AVI); after C4HSL addition: $1.07 \pm 0.02$ (0.1 $\mu$ M;

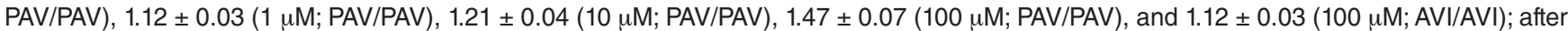
C12HSL addition: $1.09 \pm 0.02(0.1 \mu \mathrm{M}$; PAV/PAV), $1.23 \pm 0.04$ ( $1 \mu \mathrm{M} ; \mathrm{PAV} / \mathrm{PAV}), 1.48 \pm 0.04(10 \mu \mathrm{M} ; \mathrm{PAV} / \mathrm{PAV}), 1.64 \pm 0.04$ (100 $\mu \mathrm{M} ; \mathrm{PAV} / \mathrm{PAV})$, and $1.14 \pm 0.03(100 \mu \mathrm{M} ; \mathrm{AVI} / \mathrm{AVI}) .{ }^{*} P<0.05,{ }^{* *} P<0.01$ as denoted by brackets; ${ }^{\#} P<0.01$ vs. PBS; NS, no significance vs. PBS. All statistical analyses were performed using an ANOVA model with Tukey-Kramer post hoc analysis.

tion was elicited with C12HSL $(10 \mu \mathrm{M}$ and $100 \mu \mathrm{M})$ (Figure 3, E and $\mathrm{F}$ ) and was blocked by anti-TAS2R38 siRNAs (Supplemental Figure 12, F and G). Notably, at equal concentrations, C12HSL generated more NO than C4HSL (Figure 3, C and D), in agreement with the finding that it has slightly greater potency as a T2R38 agonist (Figure 2G; note that the C4HSL concentration used in Figure 2 is 2-fold greater than the C12HSL concentration). Diluted Pseudomonas-conditioned medium (6.25\%) also activated an NO response (Supplemental Figure 13). No NO production was observed when epithelial cells were stimulated with diluted conditioned medium from a strain that does not produce C4HSL or C12HSL (PAO-JP2) (56), or when cells were stimulated with Pseudomonas LPS $(10 \mu \mathrm{g} / \mathrm{ml})$ or FSL-1 $(1 \mu \mathrm{g} / \mathrm{ml})$ (Supplemental Figure $13)$, suggesting that this is a specific response to quorum-sensing molecules. We observed similar DAF-FM fluorescence increases when acutely dissociated sinonasal ciliated epithelial cells were stimulated with the T2R38 agonists PTC, NaSCN, and acetylthiourea (ATU) (52), as well as C4HSL (Supplemental Figure 14). The combination of these results with those shown in Supplemental Figure 7 strongly suggests that the responses we observe in ALI cultures reflect physiological responses in vivo.

T2R38 increases mucociliary transport velocity. MCC is the primary physical defense of the respiratory system (57). Because NO has been previously linked to elevation of $\operatorname{CBF}(8,18,19)$, we examined whether Pseudomonas-conditioned medium or purified AHLs stimulated CBF in a T2R38-dependent and/or NO-dependent fashion. Following a frequently used convention $(13,58,59)$, changes in $\mathrm{CBF}$ were normalized to the basal rate and reported as a ratio of 

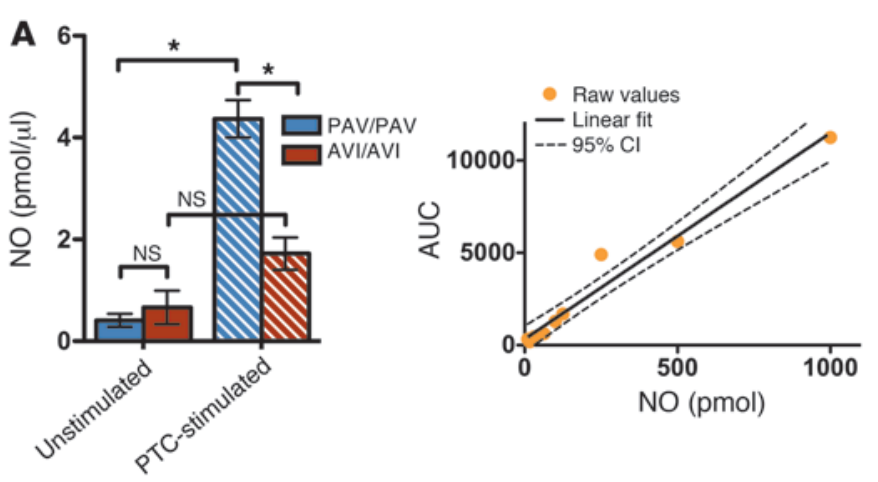

B
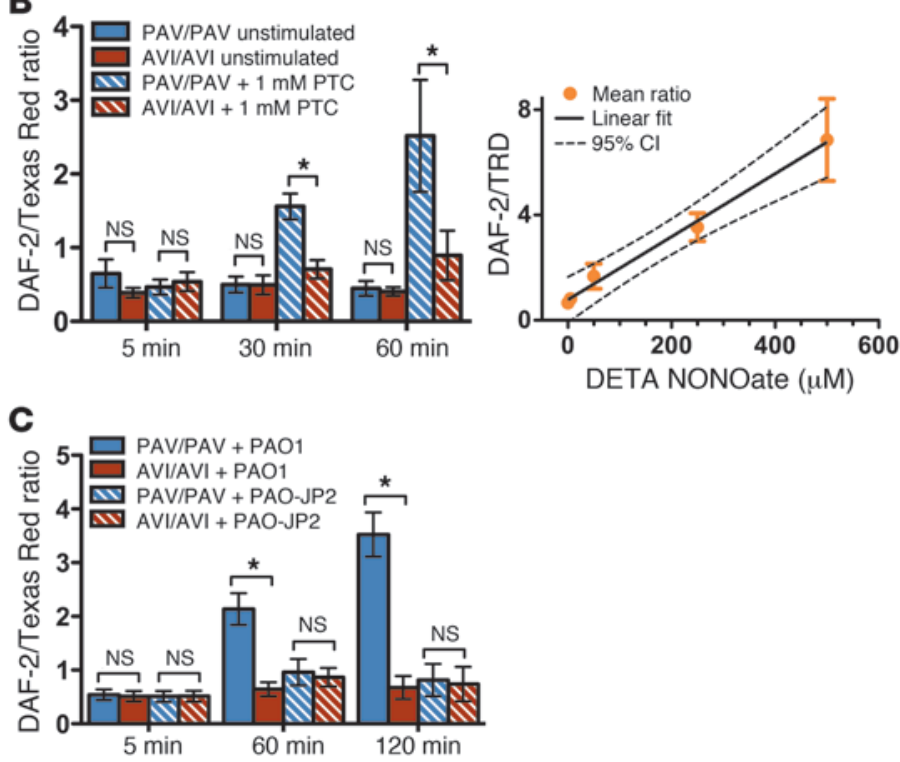

\section{Figure 6}

Human sinonasal ALI cultures exhibit T2R38-dependent apical NO diffusion. (A) Left: NO metabolites were quantified from ASL (1 stimulated and 1 unstimulated culture used from 4 PAV/PAV and $3 \mathrm{AVI} / \mathrm{AVI}$ patients each). Right: Calibration using known $\mathrm{NaNO}_{3}$ standards. (B) Left: Fluorescence ratios of DAF-2 and Texas Red Dextran (TRD) were used to measure NO secretion. There was no change in ratios for unstimulated PAV/PAV or AVI/AVI cultures (solid bars; $0.65 \pm 0.19[\mathrm{PAV} / \mathrm{PAV}]$ and $0.39 \pm 0.07[\mathrm{AVI} / \mathrm{AVI}]$ at 5 minutes, $0.50 \pm 0.11[\mathrm{PAV} / \mathrm{PAV}]$ and $0.50 \pm 0.10[\mathrm{AVI} / \mathrm{AVI}]$ at 30 minutes, and $0.45 \pm 0.10$ [PAV/PAV] and $0.41 \pm 0.06[\mathrm{AVI} / \mathrm{AVI}]$ at 60 minutes). In contrast, PAV/PAV and AVI/AVI cultures had marked differences after PTC stimulation $(0.46 \pm 0.10$ [PAV/PAV] and $0.54 \pm 0.1[\mathrm{AVI} / \mathrm{AVI}]$ at 5 minutes, $1.55 \pm 0.17$ [PAV/PAV] and $0.71 \pm 0.1[\mathrm{AVI} / \mathrm{AVI}]$ at 30 minutes, and $2.52 \pm 0.76[\mathrm{PAV} / \mathrm{PAV}]$ and $0.90 \pm 0.3[\mathrm{AVI} / \mathrm{AVI}]$ at 60 minutes). Right: Addition of $0,5,50$, 250, and $500 \mu \mathrm{M}$ DETA NONOate resulted in a linear increase in the DAF-2/TRD ratio. (C) Exposure to WT, but not AHL-deficient, Pseudomonas induced T2R38-dependent NO secretion. DAF-2/ Texas Red ratios after exposure to PAO1 were $0.5 \pm 0.1$ (PAV/PAV) and $0.5 \pm 0.1$ (AVI/AVI) at 5 minutes, $2.1 \pm 0.3$ (PAV/PAV) and $0.6 \pm 0.1(\mathrm{AVI} / \mathrm{AVI})$ at 60 minutes, and $3.5 \pm 0.4$ (PAV/PAV) and $0.7 \pm 0.2(\mathrm{AVI} / \mathrm{AVI})$ at 120 minutes. Ratios after exposure to PAO$\mathrm{JP} 2$ were $0.5 \pm 0.1(\mathrm{PAV} / \mathrm{PAV})$ and $0.5 \pm 0.1(\mathrm{AVI} / \mathrm{AVI})$ at $5 \mathrm{~min}-$ utes, $1.0 \pm 0.2(\mathrm{PAV} / \mathrm{PAV})$ and $0.9 \pm 0.1(\mathrm{AVI} / \mathrm{AVI})$ at 60 minutes, and $0.8 \pm 0.3(\mathrm{PAV} / \mathrm{PAV})$ and $0.7 \pm 0.3(\mathrm{AVI} / \mathrm{AVI})$ at 120 minutes. ${ }^{*} P<0.05$, ANOVA with Tukey-Kramer analysis. stimulated/basal frequencies. No differences in baseline or ATPstimulated CBF were observed between cultures with functional or nonfunctional T2R38 receptors, and none of the inhibitors used in this study had a significant effect on basal CBF (Supplemental Figure 15). We found that dilute Psendomonas biofilm-conditioned medium increased CBF in sinonasal ALI cultures in a dosedependent fashion (Figure 4A). This stimulation was significantly greater in cultures with the functional (PAV/PAV) T2R38 receptors than in cultures with nonfunctional receptors (AVI/AVI) or heterozygous receptors (PAV/AVI) (Figure 4, B and C). In PAV/PAV cultures, $\mathrm{CBF}$ elevation in response to $6.25 \%$ Psendomonas medium was blocked by inhibition of either PLC $\beta 2$ or NOS (Supplemental Figure 16). Conditioned medium from PAO1 planktonic cultures likewise activated a T2R38-dependent increase in CBF at dilute concentrations (Supplemental Figure 17A), while medium from PAO-JP2 (C4HSL- and C12HSL-deficient) cultures had a minimal effect on CBF and exhibited no difference between PAV/PAV and AVI/AVI cultures (Supplemental Figure 17B). As a control, culture supernatant from a flagellin mutant (Sad36; flgk) (60) yielded similar results to wild-type PAO1 medium (Supplemental Figure 17C). We hypothesized that T2R38 functions to detect AHLs secreted by Pseudomonas and other gram-negative bacteria into the airway surface liquid (ASL) environment. When PAO1 bacteria were resuspended in PBS with $0.5 \mathrm{mM}$ glucose (to simulate ASL), the resulting conditioned PBS (CPBS) stimulated increases in $\mathrm{CBF}$ in as little as 30 minutes (Supplemental Figure 18, A-C). PAO1 CPBS that was dialyzed to remove low-molecular-weight $(<3.5 \mathrm{kDa})$ compounds as well as PAO-JP2-CPBS had no effect on CBF, while the Sad36 CPBS retained stimulatory capacity (Supplemental Figure 18, D-F). Not surprisingly, C4HSL and C12HSL similarly induced a T2R38-dependent increase in CBF (Figure 4, D and E). AHLstimulated CBF increase was blocked by L-NAME, but not by the inactive D-NAME (Supplemental Figure 19), as well as by various inhibitors of NO signaling, including the soluble guanylyl cyclase inhibitor LY83583 and the protein kinase G inhibitors KT5823 and H8 (Supplemental Figure 20). These findings demonstrate an important role for T2R38 in NO-dependent ciliary responses to secreted factors from gram-negative bacteria.

To confirm that an increase in CBF yielded an increase in MCC, we measured the transport velocity of fluorescent microspheres on sinonasal ALI cultures. Cultures were washed with PBS to remove large mucus particles, and $2 \mu \mathrm{m}$ fluorescent microspheres were overlaid onto the culture. Bead transport was imaged using 2- or 5-second exposures (depending on the baseline transport rate of each culture). The length of the imaged streak reflected the distance traveled during the exposure period and thus could be used to extrapolate relative velocity changes within each experiment. 10 frames were recorded at baseline, and a subsequent 10 frames were recorded 3 minutes after the addition of Psendomonas conditioned medium to a final concentration of $6.25 \%$ (the 

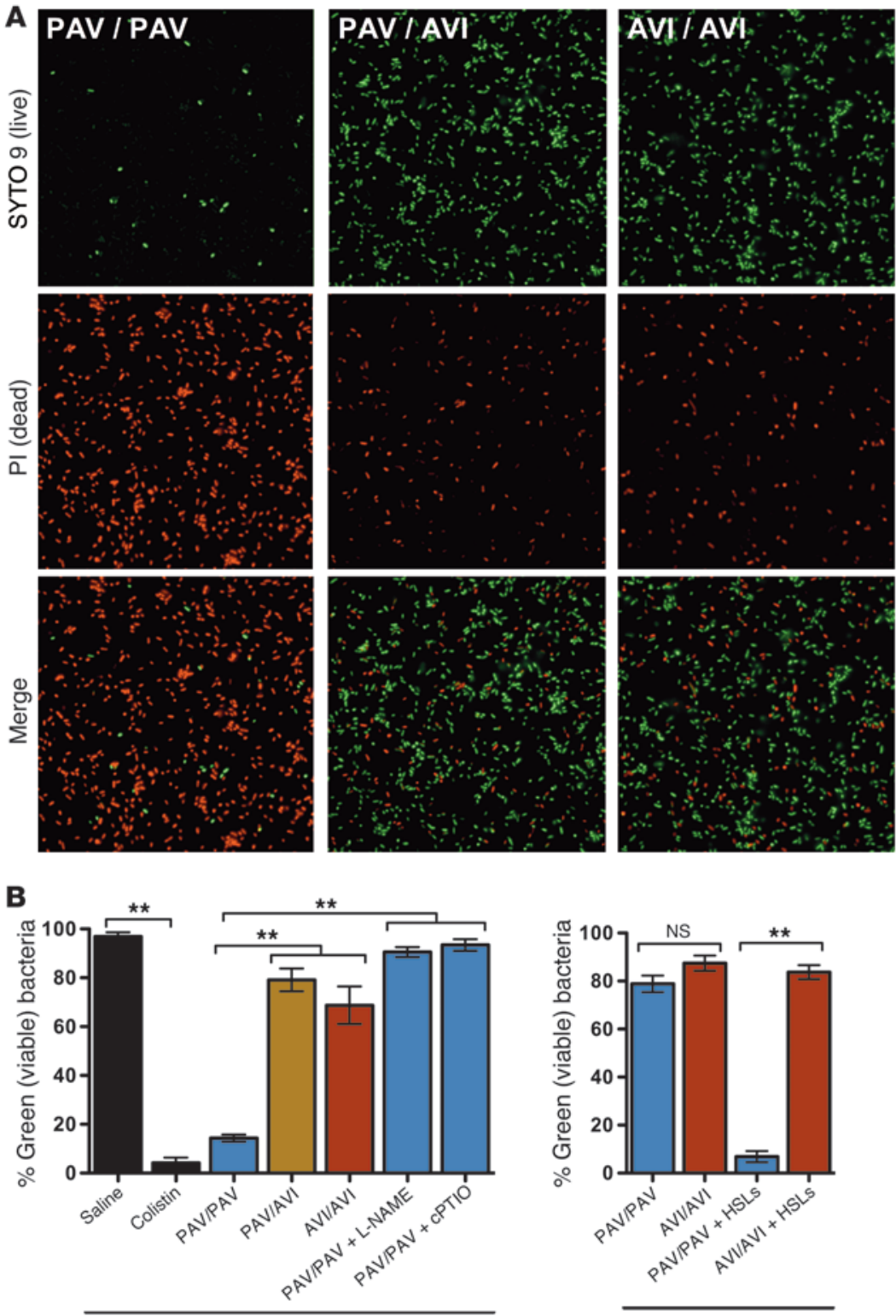

$\mathrm{PAO} 1$

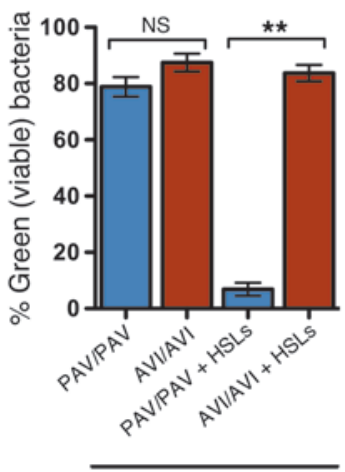

PAO-JP2

\section{Figure 7}

T2R38 is required for maximal epithelial killing of $P$. aeruginosa. (A) PAO1 removed from cultures after 2 hours of exposure showed increased propidium iodide fluorescence (indicating bacterial cell permeability) and decreased SYTO 9 fluorescence in bacteria exposed to PAV/PAV cultures vs. PAV/AVI and $A V I / A V I$ cultures. Original magnification, $\times 60$. (B) Left: Percentage of green (viable) PAO1 after exposure to saline (no epithelial cells; negative control; $97 \% \pm 2 \%$ ), colistin (no epithelial cells; positive control; $4 \% \pm 2 \%$ ), PAV/PAV cultures $(14 \% \pm 1 \%)$, PAV/AVI cultures $(80 \% \pm 5 \%), A V I / A V I$ cultures $(70 \% \pm 5 \%)$, PAV/ PAV cultures plus L-NAME $(90 \% \pm 2 \%)$, PAV/PAV cultures plus cPTIO $(93 \% \pm 2 \%)$, and washed PAV/ PAV cultures $(45 \% \pm 3 \%)$. Right: Percentage of viable PAO-JP2 from separate experiments after exposure to PAV/PAV and AVI/AVI cultures $(79 \% \pm 3 \%$ and $87 \% \pm 3 \%$, respectively) as well as PAV/PAV and AVI/ AVI cultures plus $10 \mu \mathrm{M}$ each C4HSL and $\mathrm{C} 12 \mathrm{HSL}$ (7\% $\pm 2 \%$ and $84 \% \pm 3 \%$, respectively). ${ }^{*} P<0.05$, ${ }^{\star \star} P<0.01$, ANOVA with Bonferroni analysis. field of view was unchanged; the 3-minute wait was necessary to ensure that the microspheres had settled after fluid addition). Frames from experiments using cultures from functional (PAV/ PAV) and nonfunctional (AVI/AVI) T2R38 receptors are shown in Figure 5A. Cultures homozygous for the functional form of the T2R38 receptor exhibited an approximately 1.9-fold increase in streak length (reflecting transport velocity) following application of Psendomonas conditioned medium, while nonfunctional T2R38 cultures exhibited minimal increases, as did functional cultures treated with L-NAME or the NO scavenger carboxy-PTIO (Figure 5B). Additionally, CPBS from PAO-1, PAO-JP2, and Sad36 strains were tested in this assay. We found that PAO-1 and Sad36 CPBS activated mucociliary transport in PAV/PAV but not AVI/AVI cultures (Figure 5C). In contrast, CPBS from the AHL-deficient PAOJP2 did not significantly increase transport velocity in cultures of either genotype (Figure 5C). When C4HSL and C12HSL were directly tested in this assay (Figure 5D), we found that C12HSL was more potent than C4HSL, as C12HSL significantly increased mucociliary transport at a concentration $(1 \mu \mathrm{M})$ one order of magnitude below the concentration of C4HSL $(10 \mu \mathrm{M})$ required to stimulate a significant increase in transport. This result agrees with the general trend observed in intracellular $\mathrm{NO}$ production in response to C4HSL and C12HSL (Figure 3, C and E). These data demonstrate that detection of bacterial quorum-sensing molecules by T2R38 results in the activation of intracellular $\mathrm{Ca}^{2+}$ and NO signaling pathways, resulting in enhanced MCC.

$T 2 R 38$ activation in upper respiratory epithelial cells results in NO diffusion into the ASL. The antibacterial properties of NO help maintain the sterility of the sinonasal airways (61). Thus, in addition to identifying the intracellular pathways downstream of NO generation, we tested if T2R38-induced NO production resulted in detectible diffusion of $\mathrm{NO}$ across the apical membrane into the 


\section{Table 1}

Functional and nonfunctional allele frequency

\begin{tabular}{|c|c|c|c|c|c|c|c|c|c|c|c|c|}
\hline & \multicolumn{3}{|c|}{$T A S 2 R 38^{A}$} & \multicolumn{3}{|c|}{ TAS2R1gв } & \multicolumn{3}{|c|}{ TAS2R30C } & \multicolumn{3}{|c|}{$T A S 2 R 46^{D}$} \\
\hline & AVI/AVI & AVI/PAV & PAV/PAV & $A / A$ & $A / G$ & G/G & $A / A$ & $A / C$ & $\mathrm{C} / \mathrm{C}$ & $\mathrm{C} / \mathrm{C}$ & $\mathrm{C} / \mathrm{T}$ & $\mathrm{T} / \mathrm{T}$ \\
\hline No growth & 13 & 11 & 11 & 4 & 13 & 3 & 3 & 17 & 5 & 13 & 11 & 2 \\
\hline $\begin{array}{l}\text { Gram-negative } \\
\text { P. aeruginosa }\end{array}$ & $\begin{array}{l}7 \\
5\end{array}$ & $\begin{array}{c}14 \\
9\end{array}$ & $\begin{array}{l}0 \\
0\end{array}$ & $\begin{array}{l}5 \\
4\end{array}$ & $\begin{array}{l}5 \\
5\end{array}$ & $\begin{array}{l}5 \\
2\end{array}$ & $\begin{array}{l}5 \\
2\end{array}$ & $\begin{array}{l}7 \\
7\end{array}$ & $\begin{array}{l}5 \\
4\end{array}$ & $\begin{array}{c}10 \\
7\end{array}$ & $\begin{array}{l}6 \\
5\end{array}$ & $\begin{array}{l}0 \\
0\end{array}$ \\
\hline Total (no growth + gram-negative) & 20 & 25 & 11 & 9 & 18 & 8 & 8 & 24 & 10 & 23 & 17 & 2 \\
\hline
\end{tabular}

ATyped for polymorphisms A49P, V262A, and I296V (rs713598, rs1726866 and rs 10246939; see Results and Methods). $\chi^{2}$ comparisons: 10.3 ( $P$ < 0.0058 ), no growth vs. gram-negative; $7.1(P<0.029)$, no growth vs. $P$. aeruginosa. BPutative quinine receptor, typed for $r$ s 10772420 . Receptors containing the A allele have a higher level of function than receptors containing the $\mathrm{G}$ allele (93). $\chi^{2}$ comparisons: $3.5(P<0.17)$, no growth vs. gram-negative; $1.2(P<0.54)$, no growth vs. $P$. aeruginosa. CFormerly known as TAS2R47, typed for $r$ s2708381. $\chi^{2}$ comparisons: $3.3(P<0.20)$, no growth vs. gram-negative; $0.76(P<0.68)$, no growth vs. $P$. aeruginosa. ${ }^{D}$ Typed for $r$ 2599404. $\chi^{2}$ comparisons: $1.6(P<0.46)$, no growth vs. gram-negative; $1.0(P<0.60)$, no growth vs. $P$. aeruginosa.

ASL of sinonasal cultures. NO diffusion into the ASL was quantified by measuring NO-derived byproducts in $20 \mu \mathrm{l}$ of ASL from cultures stimulated with $1 \mathrm{mM}$ PTC (or PBS alone) for $20 \mathrm{~min}-$ utes using reductive chemistries coupled with chemiluminescence detection of $\mathrm{NO}$ (Figure 6A). ASL levels of NO metabolites (nitrate, nitrite, $\mathrm{N}$-nitroso, and iron-nitrosyl adducts as well as low-molecular-weight and protein S-nitrosothiol adducts) were not different in unstimulated PAV/PAV and AVI/AVI cultures $(0.41 \pm 0.13 \mathrm{pmol} /$ $\mu \mathrm{l}$ for PAV/PAV and $0.66 \pm 0.33$ for AVI/AVI; $P=\mathrm{NS})$. However, the increase observed in PTC-stimulated PAV/PAV cultures was more than 2-fold larger than that observed in AVI/AVI cultures (Figure 6A). In PTC-stimulated cultures, NO metabolites in the ASL over 20 minutes were $4.37 \mathrm{pmol} / \mu \mathrm{l}(\mathrm{PAV} / \mathrm{PAV} ; P<0.001 \mathrm{vs}$. unstimulated $\mathrm{PAV} / \mathrm{PAV}$ ) and $1.72 \mathrm{pmol} / \mu \mathrm{l}(\mathrm{AVI} / \mathrm{AVI} ; P<0.01$ vs. stimulated $\mathrm{PAV} /$ PAV; $P=$ NS vs. unstimulated AVI/AVI).

We also utilized a fluorescence-based assay to measure NO diffusion into the ASL. The apical surface of cultures was overlaid with a small volume $(10 \mu \mathrm{l})$ of PBS with or without $1 \mathrm{mM}$ PTC containing 2 spectrally distinct cell-impermeant dyes, the NOsensitive 4,5-diaminofluorescence diacetate (DAF-2) and the NOinsensitive Texas Red dextran (10,000 kDa MW); a radiometric measurement was used to eliminate any artifacts due to expansion or contraction of ASL volume. The ratio of DAF-2/Texas Red fluorescence was recorded by fluorescence microscopy after 5-, 30-, and 60 -minute incubations at $37^{\circ} \mathrm{C}$. The greater increase in DAF-2/ Texas Red ratio in PTC-stimulated but not unstimulated PAV/PAV cultures compared with AVI/AVI cultures (Figure 6B) further demonstrates that NO diffused into the ASL in a T2R38-dependent manner. We utilized this assay to determine whether exposure to Pseudomonas strains and their secretions was sufficient to cause epithelial cells to produce NO with subsequent diffusion into the ASL. Log-phase cultures of PAO1 and PAO-JP2 were resuspended in PBS and placed on the ALI surface in the presence of DAF-2 and Texas Red. Exposure to PAO1 over 60-120 minutes, but not PAOJP2, resulted in NO diffusion into the ASL in PAV/PAV but not AVI/AVI cultures (Figure 6C), suggesting that this is a physiological response to bacterial secretion of AHLs into the ASL. Together these data indicate that PAV/PAV individuals may have enhanced sinonasal NO production compared with PAV/AVI and AVI/AVI individuals during respiratory challenge with Pseudomonas or other gram-negative bacteria.

T2R38-dependent NO production directly contributes to epithelial innate defense. We developed an assay to test whether T2R38-activated NO production could directly result in or enhance epithelial bacteri- cidal activity. ALI cultures were inoculated with planktonic (0.1 OD) Pseudomonas (PAO-1) in conditioned saline (60 minutes; as described in the Methods). After 2 hours of exposure to the ALI culture, bacteria were stained with a live/dead (green/red) stain to determine the percentage of viable (green) cells. As a control, bacteria in saline that were not exposed to an ALI culture exhibited nearly $100 \%$ green fluorescence, whereas bacteria exposed to the antibiotic colistin exhibited nearly $100 \%$ red fluorescence (Supplemental Figure 21). PAV/PAV cultures exposed to strain PAO-1 exhibited marked bacterial killing (Figure 7A), while PAV/ AVI and AVI/AVI cultures exhibited severely reduced bactericidal activity (Figure 7A). This bacterial killing required NO, as it was inhibited by basolateral L-NAME pre-treatment or the addition of cPTIO (Supplemental Figure 22, A and B). Significantly reduced killing was also observed when the AHL-deficient strain PAO-JP2 was used on PAV/PAV or AVI/AVI cultures, but the addition of C4HSL $(10 \mu \mathrm{M})$ and C12HSL $(10 \mu \mathrm{M})$ to PAO-JP2-conditioned saline restored the potent antibacterial activity of PAV/PAV but not AVI/AVI cultures (Supplemental Figure 22C). These results are shown in Figure 7B. Taken in total, our data suggest that individuals with the PAV/PAV TAS2R38 genotype have an increased capacity to detect, clear, and kill sinonasal gram-negative bacteria compared with PAV/AVI and AVI/AVI individuals, which should manifest in less gram-negative infections in PAV/PAV individuals.

Reduced T2R38 functionality correlates with gram-negative airway infection. To determine whether the above in vitro results have clinical significance, TAS2R38 was genotyped in patients who had undergone sinonasal surgery for various indications including chronic rhinosinusitis and pituitary pathology, for whom we had both tissue samples as well as microbiological laboratory reports from sinonasal swabs obtained at the time of surgery. We selected samples for TAS2R38 genotyping that the microbiology report had described as either "no growth/normal respiratory flora/coagulase-negative staph" or isolated gram-negative bacteria. In this context, coagulase-negative staph is considered either a commensurate nasal microbe or a contaminant from the nasal vestibule. We found a significant difference in the distribution of TAS2R38 functional and nonfunctional allele frequency between patients who exhibited no bacterial growth or normal respiratory flora compared with patients who tested positive for gram-negative cultures, including $P$. aeruginosa (Table 1 ). Among the patients with sinonasal gram-negative bacteria, none were homozygous for the functional (PAV/PAV) form of TAS2R38 $\left(P<0.0058\right.$ by $\chi^{2}$ analysis); the results were also significant when patients with no growth 
were compared with patients with $P$. aeruginosa cultures $(P<0.029)$. No significant differences were observed between the 2 groups when polymorphisms for 3 other bitter receptor genes were examined, namely TAS2R19, TAS2R30 (formerly known as TAS2R47), and TAS2R46 (Table 1). Some genotypes could not be unequivocally determined using allele-specific primers and probes, probably due to the presence of copy number variants (62), and thus not all genotypes were reported for each patient. Detailed information about these patients is reported in Supplemental Table 1. These genetic data support the clinical relevance of the in vitro physiological data described above, which suggest that T2R38 plays a role in respiratory epithelial defense against gram-negative bacteria, including the common respiratory pathogen Pseudomonas.

\section{Discussion}

Our findings indicate that the GPCR T2R38, originally identified as a bitter taste receptor, is expressed in HSECs, where it triggers a rapid inter-kingdom signaling response bridging microbial secretions and the human upper airway epithelium, making it an integral component of upper respiratory early response defenses. When stimulated by AHLs, T2R38 elicits calcium-dependent NO production that increases $\mathrm{CBF}$ and mucus clearance. Additionally, this NO diffuses into the airway and contributes to innate antimicrobial effects. We further demonstrate that upper airway epithelial cells from individuals with 1 or 2 nonfunctional T2R38 alleles have significantly blunted NO and ciliary responses following exposure to gram-negative quorum-sensing molecules, and that these individuals are more likely to be infected with gram-negative bacteria such as $P$. aeruginosa than those with 2 functional receptor alleles.

While initially it may be surprising that AHLs are direct T2R38 agonists that are quite structurally different from the classical thiolactone T2R38 agonists (such as PTC, propylthiouracil, ATU, etc.), there is precedence for a large structural diversity among the compounds that activate T2R38 as well as other T2Rs in heterologous expression systems (52). Examples of the diversity of T2R38 agonists include the thiolactones, the muscarinic antagonist diphenidol, the D-ring lactone limonin, and the alkaloid yohimbine. This suggests the possibility of multiple binding sites on T2R38, a model that has been proposed to explain the broad responsiveness of some T2Rs (63) as well as other gustatory receptors such as the T1R2/3 sweet receptor (64). As described above, the PAV and AVI polymorphisms likely affect the structure of the receptor and its ability to be activated, not agonist binding per se, explaining why the AVI polymorphism can block the response to a variety of T2R38 agonists including AHLs. Further molecular characterization is needed to understand the interaction between AHLs and T2R38.

Long-term exposure to Pseudomonas AHLs has been shown to have toxic effects in some airway cell lines (65). A reduced ability to rapidly detect and clear these molecules in PAV/AVI and AVI/AVI individuals may have profound disease implications. While AHL concentrations of more than $100 \mu \mathrm{M}$ are only found in mature biofilms, the levels found in planktonic growth (pre-biofilm) cultures are still quite high and within the range of detection we observe in this study. C12HSL levels have been reported to be between $200 \mathrm{nM}$ (early log phase) and $5 \mu \mathrm{M}$ (early stationary phase) in planktonic laboratory cultures (66-68), while the level of C4HSL has been calculated at $10 \mu \mathrm{M}$ in early stationary phase planktonic cultures (66). The T2R38-dependent detection of individual AHLs in the low $\mu \mathrm{M}$ range (Figure 5) as well as the detection of secreted AHLs in CPBS after only 30 minutes (Supplemental Figure 18) suggests that the T2R38 system serves as a mechanism to prevent infection and biofilm formation through early detection of AHLs and subsequent eradication of bacteria. This hypothesis is supported by the clinical data presented, which demonstrates underrepresentation of individuals homozygous for the functional T2R38 in patients harboring gram-negative sinonasal bacteria and warrants prospective studies to demonstrate the contribution of this pathway and these genetics in upper airway infection.

It is interesting to note that the nonfunctional haplotype (AVI) is not found in non-human primate species, which suggests that this polymorphism appeared following human divergence from our primate ancestors (41). Why both the functional and nonfunctional TAS2R38 alleles occur at high frequencies in the population remains unanswered. Indeed, it has been suggested that the "nonfunctional" allele may have an unknown function that helps to maintain it in the population (69). However, the hypothesis that T2R38 is involved in respiratory defense raises a novel hypothesis to explain the differential distribution of allele frequencies worldwide. It may be only at certain times or in certain places that specific bacteria assault the airway and secrete compounds detected by T2R38. In fact, bitter receptors change over evolutionary time more rapidly than other $G$ protein-coupled receptors (70), perhaps to adapt to changing bacterial signals. An observation that supports this hypothesis is that humans with more non-taster alleles live in the colder climates, where the evolutionary pressure for the taster genotype may be relaxed, as there are fewer pathogens than in warmer climates (71).

We observed that people with 1 functional and 1 nonfunctional allele of the T2R38 receptor (PAV/AVI) were more similar in their response to people with two nonfunctional copies (AVI/AVI), a pattern that varies from that observed for taste perception, where heterozygotes have a wide range of responses but, on average, are more like the group with two functional receptor copies (PAV/ PAV) and can readily detect PTC at low concentrations (41). The mechanisms underlying this difference between the airway cells and taste cells are not yet known, but it is possible that expressing just one nonfunctional allele leads to increases of intracellular $\mathrm{Ca}^{2+}$ that are insufficient to activate NOS following T2R38 activation. It is also possible that there is differential allele expression, with the AVI allele being overexpressed or with silencing of the PAV allele. Further work must be done to address this issue.

In conclusion, our data suggest that T2R38 genotype represents a defining characteristic in respiratory innate defense that contributes to the complex genetic and environmental interactions predisposing to upper respiratory infections. Moreover, these data provide the preclinical evidence to motivate a clinical trial to prospectively define the clinical course of patients with various T2R38 genotypes while simultaneously carefully phenotyping their response to a bitter (PTC-like) taste test. If gustatory response to PTC accurately predicts rates of sinonasal infection or response to therapy for sinonasal infection, a bitter taste test could serve as an indicator for aggressive therapy in patients with rhinosinusitis. Furthermore, increased mucociliary clearance and NO production are not specific defenses against gram-negative infection; rather, they are global antimicrobial responses. Since even individuals with nonfunctional alleles will respond to high concentrations of T2R38 agonists (29), topical application of such agonists may have therapeutic potential for multiple forms of upper respiratory infections. 


\section{Methods}

Reagents. C4HSL, SNAP, DAF-2, and cPTIO were from Cayman Chemical. Fluo-4, DAF-FM, thapsigargin, ionomycin, Texas Red dextran (10,000 $\mathrm{MW}$ ), and fluorescent microspheres were from Invitrogen. Unless indicated, other reagents were from Sigma-Aldrich. Stock solutions of PTC, C4HSL, C12HSL, Fluo-4, DAF-2, DAF-FM, cPTIO, SNAP, thapsigargin, and ionomycin were made at 1:1,000 dilution in DMSO, with working solutions made fresh daily. The working SNAP solution (in PBS) was kept on ice in the dark and warmed immediately before use. NaSCN, denatonium, thujone, ATU, and Texas Red dextran stocks were dissolved directly in PBS. LPS from $P$. aeruginosa serotype 10 were purchased from Sigma-Aldrich, and FSL-1 was a gift from D. LaRosa (University of Pennsylvania, Philadelphia, Pennsylvania, USA). ELISAs for $\beta$-defensin and IL-8 were purchased from Adipo Biosciences and Pierce Biotechnology, respectively, and performed according to the manufacturer's instructions. A Luminex 18-plex kit was used as previously described (72).

Experimental solution compositions. Physiological experiments were performed with Dulbecco's PBS (DPBS; containing $1.8 \mathrm{mM} \mathrm{Ca}^{2+}$ ) on the apical side of the cultures. The basolateral side was bathed in modified HEPESbuffered HBSS containing $1 \times$ MEM amino acids (Invitrogen) to provide a source of arginine $(\sim 0.6 \mathrm{mM})$ for $\mathrm{NO}$ production. DPBS contained (in $\mathrm{mM}) 138 \mathrm{NaCl}, 2.7 \mathrm{KCl}, 1.5 \mathrm{KH}_{2} \mathrm{PO}_{4}, 8 \mathrm{Na}_{2} \mathrm{HPO}_{4}, 1.8 \mathrm{CaCl}_{2}$, and $1.5 \mathrm{MgCl}_{2}$, with $\mathrm{pH}$ adjusted to approximately 7.2 (to prevent any $\mathrm{Ca}^{2+}$ precipitation due to the high phosphate concentrations in PBS). HBSS contained (in mM) $137 \mathrm{NaCl}, 5 \mathrm{KCl}, 0.4 \mathrm{KH}_{2} \mathrm{PO}_{4}, 0.3 \mathrm{Na}_{2} \mathrm{HPO}_{4}, 5.5$ glucose, $1.8 \mathrm{CaCl}_{2}$, $1.5 \mathrm{MgCl}_{2}, 10 \mathrm{HEPES}, \mathrm{pH}$ 7.4. Experiments performed in the absence of extracellular $\mathrm{Ca}^{2+}$ utilized the above solutions with no added $\mathrm{Ca}^{2+}$ and containing $1 \mathrm{mM}$ EGTA.

Sinonasal ALI cultures. Patients were recruited from the Division of Rhinology in the Department of Otorhinolaryngology - Head and Neck Surgery at the University of Pennsylvania, and from the Philadelphia Veterans Affairs Medical Center, with full study approval from the institutional review boards at both institutions. Informed consent was obtained during the pre-operative clinic visit or in the pre-operative waiting room. Selection criteria for recruitment were patients undergoing sinonasal surgery. Exclusion criteria included a history of systemic diseases such as Wegner's granulomatosis, sarcoidosis, cystic fibrosis, immunodeficiencies, and use of antibiotics, oral corticosteroids, or antibiologics (e.g., Xolair) within 1 month of surgery. Sinonasal mucosal specimens were acquired from residual clinical material obtained during sinonasal surgery and transported to the laboratory in saline placed on ice. ALI cultures were established from HSECs from enzymatically dissociated human tissue as previously described $(73,74)$ and grown to confluence in tissue culture flasks $\left(75 \mathrm{~cm}^{2}\right)$ with proliferation medium consisting of DMEM/Ham's F-12 and bronchial epithelial basal medium (BEBM; Clonetics) supplemented with $100 \mathrm{U} / \mathrm{ml}$ penicillin and $100 \mu \mathrm{g} / \mathrm{ml}$ streptomycin for 7 days. Cells were then trypsinized and seeded on porous polyester membranes $\left(6 \sim 7 \times 10^{4}\right.$ cells per membrane) in cell culture inserts (Transwell-clear, 12 -mm diameter, $0.4-\mu \mathrm{m}$ pores; Corning) coated with $100 \mu \mathrm{l}$ of coating solution (BSA [0.1 mg/ml; Sigma-Aldrich], type I bovine collagen [30 $\mu \mathrm{g} /$ $\mathrm{ml}$; BD Biosciences], and fibronectin [10 $\mu \mathrm{g} / \mathrm{ml}$; BD Biosciences] in LHC basal medium [Invitrogen]) and left in a tissue culture laminar flow hood overnight. Five days later the culture medium was removed from the upper compartment and the epithelium was allowed to differentiate by using the differentiation medium consisting of 1:1 DMEM (Invitrogen) and BEBM (Clonetics; Cambrex) with the Clonetics complements for hEGF (0.5 ng/ $\mathrm{ml})$, epinephrine $(5 \mathrm{~g} / \mathrm{ml})$, BPE $(0.13 \mathrm{mg} / \mathrm{ml})$, hydrocortisone $(0.5 \mathrm{~g} / \mathrm{ml})$, insulin $(5 \mathrm{~g} / \mathrm{ml})$, triiodothyronine $(6.5 \mathrm{~g} / \mathrm{ml})$, and transferrin $(0.5 \mathrm{~g} / \mathrm{ml})$, supplemented with $100 \mathrm{U} / \mathrm{ml}$ penicillin, $100 \mathrm{~g} / \mathrm{ml}$ streptomycin, $0.1 \mathrm{nM}$ retinoic acid (Sigma-Aldrich), and 10\% FBS (Sigma-Aldrich) in the basal compartment. Human bronchial epithelial cells (Lonza) were similarly cultured as previously described $(75,76)$. Microbiology swabs were processed by the clinical microbiology lab using both blood agar as well as MacConkey agar for isolation of gram-negative bacteria.

Bacterial culture. For biofilm-conditioned medium, P. aeruginosa strain PAO1 cultures were grown for 3 days in 24-well plates, then the resultant medium was centrifuged at $2,000 \mathrm{~g}$ for 15 minutes at room temperature and filtered through a $0.2-\mathrm{mM}$ filter. The resultant conditioned medium was then adjusted to an $\mathrm{OD}_{655}=0.35(77)$ when blanked against LB. Planktonic growth cultures of WT PAO1, PAO-JP2 ( $\left.\Delta l a s I, \Delta r b l i ; \mathrm{Tc}^{\mathrm{r}}, \mathrm{HgCl}_{2}{ }^{\mathrm{r}}\right)(56)$, and $\operatorname{Sad} 36$ ([flgK]::Tn5B30[TC $\left.\left.{ }^{\mathrm{r}}\right]\right)(60)$ were grown for 12 hours at $37^{\circ} \mathrm{C}$ with shaking in LB (PAO1) or LB plus $15 \mu \mathrm{g} / \mathrm{ml}$ tetracycline (PAO-JP2 and Sad36). Conditioned medium was prepared as described above. CPBS was made by taking a 12 -hour overnight culture, diluting it to $0.1 \mathrm{OD}$ (log phase), and incubating at $37^{\circ} \mathrm{C}$ with shaking for 90 minutes in LB. Bacteria were then centrifuged, washed 3 times with PBS plus $0.5 \mathrm{mM}$ glucose, resuspended at $0.1 \mathrm{OD}$ in the same solution, and incubated at $37^{\circ} \mathrm{C}$ with shaking. This glucose concentration was chosen because physiological ASL glucose concentrations have been demonstrated to be approximately $10 \%$ of serum glucose, which is normally approximately $5-8 \mathrm{mM}(78-81)$. Aliquots of this solution were taken at 30 minutes, 2 hours, and 6 hours and then centrifuged and filter sterilized as described above. Bacteria did not grow robustly under these conditions, as the initial ODs $(0.12 \pm 0.03$, $0.10 \pm 0.02$, and $0.13 \pm 0.03$ for PAO1, PAO-JP2, and Sad36, respectively; average of 3 independent dilutions and readings) were not significantly different from the final ODs $(0.15 \pm 0.03,0.13 \pm 0.02$, and $0.16 \pm 0.01$, respectively). Dialysis of CPBS was performed for approximately 16 hours at $4{ }^{\circ} \mathrm{C}$ against an approximately 1,000 -fold excess of the same PBS solution (changed once after the first 6 hours) using a 3,500 MW cutoff dialysis membrane (Spectra/Por; Spectrum Medical Industries Inc.). As a control, undialyzed CPBS from the same cultures was incubated similarly and tested to ensure that the lack of effect of dialyzed CPBS was not due to degradation of the bacterial products.

Immunofluorescent staining and confocal microscopy. Mucosal specimens and ALI cultures appropriate for immunostaining were fixed in $4 \%$ paraformaldehyde for 20 minutes at $4{ }^{\circ} \mathrm{C}$ and subsequently washed 3 times in PBS. Primary HSEC ALI cultures were grown to maturity, washed 3 times with PBS, and fixed in $4 \%$ paraformaldehyde for 20 minutes at room temperature. The transwell permeable support was again washed 3 times with PBS prior to excision of the membrane containing the cells from its plastic supports. The membrane was immersed in Tris-buffered saline with $0.3 \%$ Triton X-100, $5 \%$ normal donkey serum, and $1 \%$ bovine serum albumin for 60 minutes at room temperature to permeabilize the plasma membrane and block nonspecific binding sites. Two primary antibodies raised from different hosts were chosen for the double immunofluorescent staining: mouse monoclonal anti- $\beta$-tubulin IV (1:1,000; Abcam) and rabbit polyclonal anti-T2R38 (1:500; Santa Cruz Biotechnologies Inc.). Visualization was carried out using Alex Fluor 488-conjugated (green) donkey antimouse IgG for tubulin IV and Alex Fluora 594 (red)-conjugated donkey anti-mouse IgG for T2R38. Both secondary antibodies (Invitrogen) were diluted at 1:500. The incubation time was overnight at $4{ }^{\circ} \mathrm{C}$ for primary antibodies and 75 minutes at room temperature for secondary antibodies, respectively. Counterstaining was done using Hoechst (blue), a nuclear dye. Confocal images were acquired using an Olympus Fluoview System at the $z$ axis step of $0.5 \mu \mathrm{m}$. A sequential scanning module was used to prevent bleed-through of fluorophores into other channels.

Profiles of red and green fluorescence (to determine the height of ciliary staining) were performed on $\mathrm{z}$ axis projections exported from Olympus Fluoview software with the RGB line profile plug-in of the MacBiophotonics (McMaster University, Hamilton, Ontario, Canada) ImageJ bundle. 
Correlation coefficients were obtained using the method of Manders (38) and the Manders coefficients plug-in of the McMaster ImageJ bundle on the raw Fluoview $\mathrm{z}$-stacks. The $\mathrm{z}$ axis step size was $0.5 \mu \mathrm{m}$; correction for the refractive index change using the paraxial approximation of the $\mathrm{n}_{2} / \mathrm{n}_{1}$ method (water 1.33/oil 1.51) resulted in an approximate pixel size of 0.44 $\mu \mathrm{m}$ (10 pixels at approximately $8 \mu \mathrm{m}$ each).

RT-PCR. ALI cultures were subjected to RNA extraction using an RNeasy Mini Kit (Qiagen). Contaminated genomic DNA was removed by treating the samples with RNase-free DNase (Qiagen) for 30 minutes at room temperature during the extraction process. cDNA synthesis was performed using $1 \mu \mathrm{g}$ RNA, Superscript III Reverse Transcriptase (Invitrogen), and random hexamers according to the manufacturers' recommendations. Negative-control templates were synthesized by omission of reverse transcriptase. PCR was performed in 50- $\mu$ l reaction tubes containing 1 unit Taq DNA Polymerase (Invitrogen), $1 \times$ PCR buffer, $1 \mu \mathrm{M}$ dNTP mix, $1.5 \mathrm{mM}$ $\mathrm{MgCl}_{2}, 1.5 \% \mathrm{DMSO}, 0.5 \mu \mathrm{M}$ forward and reverse primers, and $2 \mu \mathrm{lDNA}$. Thermal cycling conditions consisted of an initial denaturation at $94^{\circ} \mathrm{C}$ for 2 minutes, followed by 35 amplification cycles of $94^{\circ} \mathrm{C}$ for 20 seconds, $58^{\circ} \mathrm{C}$ for 40 seconds, and $72^{\circ} \mathrm{C}$ for 30 seconds. A final elongation step was performed at $72^{\circ} \mathrm{C}$ for 10 minutes.

Live-cell imaging of Fluo-4 and DAF-FM in buman ALI cultures. Fluo-4 and DAF-FM imaging was performed according to standard Methods using the 488-nm argon laser line of a Fluoview FV1000 laser scanning confocal system and IX-81 microscope ( $\times 10,0.3$ NA UPlanFLN objective; Olympus). Cells were loaded with Fluo- 4 by incubation at room temperature in the dark with apical solution containing $10 \mu \mathrm{M}$ Fluo-4-AM for approximately 90 minutes. After loading with Fluo-4, cultures were washed 3 times with DPBS, followed by incubation for 15-20 minutes to allow for de-esterification of the loaded dye. Cultures were mounted in a custom-made chamber with a \#1 coverslip bottom and basolateral solution volume of approximately $300 \mu \mathrm{l}$ (changed after each experiment). No evidence of significant photobleaching, toxicity, and/or dye extrusion was noted. Fluo-4 images were captured at approximately 5 -second intervals (scan speed $10 \mu \mathrm{s} /$ pixel; $512 \times 512$ resolution). To maximize light collection and to minimize any effects of focal drift over the time course of experiments, all experiments were performed with a fully open confocal aperture to roughly approximate wide-field conditions. No gain, offset, or gamma alterations were used. Fluo-4 fluorescence changes were normalized after subtraction of the background fluorescence, which was estimated for each experiment by measuring unloaded ALIs at identical settings. Baseline Fluo-4 fluorescence $\left(\mathrm{F}_{\mathrm{o}}\right)$ was determined by averaging the first 10 frames of each experiment.

Preliminary experiments revealed that DAF-FM loading itself caused epithelial cells to have an elevated baseline NO production, as evidenced by high background DAF-FM fluorescence at the beginning of experiments. Cells were thus loaded with DAF-FM by incubation in DPBS containing $10 \mu \mathrm{M}$ DAF-FM diacetate in PBS containing $5 \mu \mathrm{M}$ carboxy PTIO, a cellpermeant NO scavenger (on the apical side only). After 30 minutes, cultures were copiously washed (at least 5 times) with PBS to remove all traces of unloaded DAF-FM and CPTIO. This loading protocol greatly reduced starting DAF-FM fluorescence levels. Cultures were then incubated for approximately 15 minutes to allow for dye retention before imaging was performed. DAF-FM fluorescence images were acquired at 5 -second intervals using identical settings to those used for Fluo-4. Because the magnitudes of DAF-FM fluorescence changes were used to approximate NO production, care was taken to follow the loading protocol strictly to normalize dye loading, and microscope/software settings were identical for each experiment.

Transfection with siRNA. HSECs were transfected with siRNA at the time of plating on transwell filters. Cells were seeded at a density of $1 \times 10^{5}$ per transwell in medium containing 50\% LHC9 in RPMI-1640 without antibiotics together with $40 \mu \mathrm{l}$ of Opti-MEM (Invitrogen) containing preformed siRNA complexes $(100 \mathrm{nM})$ and $0.6 \mu$ l of Lipofectamine RNAi MAX. The basolateral side contained $500 \mu$ l of LHC9/RPMI- 1640 without antibiotics. Transfection reagent, siRNA, and control RNAs were all from Invitrogen, and siRNA transfection was performed according to the manufacturer's instructions. Invitrogen Stealth RNAi siRNA oligo duplexes directed against hT2R38 were CCAGAUGCUCCUGGGUAUUAUUCUU and AAGAAUAAUACCCAGGAGCAUCUGG (TAS2R38HSS108754[3_ RNAI]; siRNA 54), GGCACAUGAGGACAAUGAAGGUCUA and UAGACCUUCAUUGUCCUCAUGUGCC (TAS2R38HSS108755[3_RNAI]; SiRNA 55), and CCUACUGAUUCUGUGGCGCGACAAA and UUUGUCGCGCCACAGAAUCAGUAGG (TAS2R38HSS108756[3_RNAI]; siRNA 56). The non-targeting negative control was a stealth siRNA negative control duplex (catalog no. 12935-200). After transfection, siRNA complexes were removed after 24 hours and cells were fed with differentiation medium, as described above. The transfected cells were used after approximately 10-12 days.

Heterologous expression. The hTAS2R 38 constructs were generated according to Bufe et al. (29). In brief, the hTAS2R38 was amplified by genomic PCR from 2 PAV and AVI homozygotes. The sequence encoding the first 45 amino acids of rat SSTR-3 was amplified using PCR from rat genomic DNA. The 2 fragments were joined by overlapping PCR and inserted into pCDNA3. $1^{+}$vector. All constructs were verified by sequencing. The Ga16gust44 clone was described previously (29). HEK293 (peakRapid) cells were obtained from ATCC (CRL-2828) and cultured at $37^{\circ} \mathrm{C}$ in opti-MEM (Invitrogen) supplemented with 5\% fetal bovine serum (Invitrogen). For transient transfection, cells were seeded onto 96-well plates coated with poly-lysine at 40,000 cells per well. Cells were transfected with either version of hTAS2R38, along with Ga16-gust44. Equal amounts of each cDNA were transfected, totalling $0.2 \mu \mathrm{g}$ per well. After another 24 hours, the cells were washed with HBSS, loaded with $50 \mu \mathrm{l}$ of $3 \mu \mathrm{m}$ Fluo-4 (Invitrogen) in HBSS, and incubated for 1 hour. Then the cells were washed 3 times with HBSS and left in $50 \mu \mathrm{l}$ HBSS. The dye-loaded transfected cells in plates were placed into a Molecular Devices FlexStation III system to monitor the change in fluorescence (excitation, $488 \mathrm{~nm}$; emission, $525 \mathrm{~nm}$; cut-off, 515 $\mathrm{nm}$ ) following the addition of $50 \mu \mathrm{l}$ compound solution at twice the final concentration, 30 seconds after the start of the scan. Scanning continued for an additional 150 seconds, and data were collected every 2 seconds. Intracellular $\mathrm{Ca}^{2+}$ mobilization was quantified as the percentage of change (peak fluorescence - baseline fluorescence level; referred to herein as $\Delta \mathrm{F}$ ) from its own baseline fluorescence level (denoted as F). Peak fluorescence intensity occurred about 20 seconds after the addition of ligands. As controls, buffer alone evoked no change of fluorescence $(\Delta \mathrm{F} / \mathrm{F} \approx 0$; SEM is about $1 \%$ ). The data were expressed as the mean \pm SEM of the $\Delta \mathrm{F} / \mathrm{F}$ value of 3 independent samples.

Measurement of $\mathrm{CBF}$ and mucociliary transport velocity. Cultures were imaged using a high-speed camera (Basler 602f; 100 frames/second) attached to an inverted Leica Microscope ( $\times 20$, 0.8 NA objective) lens. CBF was measured using the Sisson-Ammons Video Analysis system (82). All experiments were performed at approximately $28^{\circ} \mathrm{C}-30^{\circ} \mathrm{C}$. Mucociliary transport velocity was measured using $2-\mu \mathrm{m}$ polystyrene fluorescent microspheres $(0.0025 \%$ by weight in $30 \mu \mathrm{l}$ ) that were added to the apical surface of the cultures after copious washing with PBS to remove mucus clumps. Beads were imaged using an inverted Nikon TE2000E epifluorescence microscope ( $\times 20,0.5$ NA PlanFluor objective) equipped with a 12-bit QImaging camera and computer running ImageJ (NIH) and $\mu$ Manager (83). To qualify for inclusion in the statistical analysis of the data, a streak had to have a visible beginning and ending within the field of view. Either an ND4 or an ND8 filter was used for bead-tracking experiments, depending on the thickness of the individual epithelial culture and resulting brightness of the beads.

Wide-field fluorescence imaging of NO secretion and particle velocity. For measurement of NO secretion, a protocol was developed in which a solution of 
Texas Red Dextran (an NO-insensitive cell-impermeant dye) and DAF-2 (an NO-sensitive cell-impermeant dye) were added in a small volume (10 $\mu \mathrm{l})$ to the apical surface of the cultures. The ratio of DAF-2/Texas Red fluorescence was recorded using wide-field epifluorescence microscopy at baseline and after incubation at $37^{\circ} \mathrm{C}$ for the indicated times. This experiment was validated by the addition of DETA-NONOate, an NO donor that releases 2 moles of NO per mole of parent molecule. DETA-NONOate was added on top of ALI cultures in $\mathrm{pH} 5$ buffer to stimulate rapid decomposition. Imaging was performed using a Nikon $\times 20,0.5$ NA PlanFluor objective and $100-\mathrm{W}$ mercury arc lamp with filters from Chroma Technologies. Green filter contained a 480/40-nm band-pass [bp] excitation filter (reported as maximum transmission wavelength/filter width at half-maximal transmission), a 505-nm dichroic mirror, and a 535/40-nm bp emission filter. The red filter set contained a 560/40-nm bp excitation filter, a 595-nm dichroic mirror, and a 630/60-nm bp emission filter.

Measurement of NO metabolites. ASL from stimulated and unstimulated cultures ( $20 \mu \mathrm{l}$ total volume for each) was collected after 20 minutes at $37^{\circ} \mathrm{C}$, frozen at $-20^{\circ} \mathrm{C}$, and protected from light until analysis. NO metabolites were evaluated by reducing metabolites, using acidifiedheated vanadium, to NO; $\mathrm{NO}$ was detected via chemiluminescence on a Sievers $280 \mathrm{NO}$ analyzer. Samples were injected into a custom-made purge container containing $0.05 \mathrm{M}$ vanadium chloride in $1 \mathrm{~N} \mathrm{HCl}$ heated to $95^{\circ} \mathrm{C}$. The reading in millivolts was compared to a standard curve generated by injecting standard solutions of nitrate. This reductive method provides information about the total levels of nitrate, nitrite, $\mathrm{N}$-nitroso, and iron-nitrosyl adducts as well as low-molecular-weight and protein S-nitrosothiol adducts.

Genetic analyses. Genomic DNA was extracted from cultured cells or sinonasal specimens following the directions of the manufacturer (QIAmp DNA Mini kit and Blood Mini kit; Qiagen). For correlation with clinical infection, sinonasal specimens of patients of mixed European descent were used since the TAS2R38 allele frequency is known for northern European descent (41). Following extraction, samples were quantified using a spectrophotometer (ND-1000; Nanodrop), using $1.5 \mu \mathrm{l}$ of extracted genomic DNA. Samples were diluted to $5 \mathrm{ng} / \mu \mathrm{l}$ and genotyped using the ABI StepOne real-time PCR system. Alleles of the TAS2R38 gene were genotyped for a variant site using allele-specific probes and primers (Applied Biosystems).

Psendomonas live-dead assay. Psendomonas from an overnight culture were diluted in $\mathrm{LB}$, grown to log phase $(\mathrm{OD}=0.1)$, then resuspended in low-salt (50\%) saline plus $0.5 \mathrm{mM}$ glucose plus $1 \mathrm{mM}$ HEPES ( $\mathrm{pH} 6.5$; gassed with $1 \% \mathrm{O}_{2}$ to minimize the immediate reaction of secreted $\mathrm{NO}$ with dissolved $\mathrm{O}_{2}$ ) and incubated with shaking for 60 minutes. These conditions were chosen to mimic physiological nasal ASL conditions (84-92). Bacteria in $30 \mu \mathrm{l}$ of this solution were placed on the apical side of the ALI and allowed to settle for approximately 10 minutes. Afterward, the majority of ASL fluid was aspirated, leaving only a minimal amount remaining. Antibiotic-free F-12 medium plus glutamate was used as the basolateral solu- tion. After a 2 -hour incubation at $37^{\circ} \mathrm{C}$ at $1 \% \mathrm{O}_{2}$, bacteria were removed from the cultures by washing with $30 \mu \mathrm{l}$ saline, which was then aspirated and placed into a chamber on a glass coverslip for viewing. Due to the thickness and geometry of the ALI filter as well as small amounts of background autofluorescence, direct viewing of bacteria on the ALI at high resolution was not feasible. Bacteria were mixed with $30 \mu \mathrm{l}$ of a $2 \times$ solution of SYTO 9 and propidium iodide (LIVE/DEAD BacLight Bacterial Viability Kit; Invitrogen) and visualized using wide-field epifluorescence filters as described above. Control experiments were performed by similarly incubating bacteria on transwell filters without cells in saline solution alone or in saline solution with $10 \mu \mathrm{g} / \mathrm{ml}$ colistin sulfate, a potent antiPseudomonas antibiotic. Staining was quantified by determining the area of positive red and green fluorescence for each image thresholding of each channel in Imagej; these values were used to calculate total fluorescent area (red + green) and the percentage of green fluorescent area, which, as live and dead cells appeared to be equal size, was assumed to equal the percentage of live (green) cells.

Statistics. All statistical analyses were performed in Excel (Student's $t$ test) and/or GraphPad Prism (Student's $t$ test, $\chi^{2}$ test, ANOVA) as indicated; $P<0.05$ was considered statistically significant. For multiple comparisons, ANOVA with the Bonferroni post hoc test was used when pre-selected pairwise comparisons were performed, ANOVA with the Tukey-Kramer post hoc test was used when all values in the data set were compared, and ANOVA with the Dunnett's post hoc test was used when all values were compared with a control value.

\section{Acknowledgments}

We thank D.M. Sabatini, P.F. Worley, A.S. Cohen, N.M. Cohen, J.C. Saunders, and Y.E. Cohen for critical reading of this manuscript. We also thank B.H. Igelwski and J.M. Schwingel for Pseudomonas strain PAO-JP2, G. O'Toole for Pseudomonas strain Sad36, and D. LaRosa for FLS-1. This work was supported by a grant from the Flight Attendants Medical Research Institute 082478 (to N.A. Cohen), a philanthropic contribution from the RLG Foundation Inc. (to N.A. Cohen), and USPHS grants P30DC011735 (to D.R. Reed), R01DC004698 (to D.R. Reed), P50DC000214 (to G.K. Beauchamp), and R01DC010842 (to P. Jiang).

Received for publication July 11, 2012, and accepted August 2, 2012.

Address correspondence to: Noam A. Cohen, Department of Otorhinolaryngology - Head and Neck Surgery, Hospital of the University of Pennsylvania, Ravdin Building 5th Floor, 3400 Spruce Street, Philadelphia, Pennsylvania 19004, USA. Phone: 215.823.5800, ext. 3892; Fax: 215.349.5977; E-mail: cohenn@ uphs.upenn.edu.
1. Sleigh MA, Blake JR, Liron N. The propulsion of mucus by cilia. Am Rev Respir Dis. 1988; 137(3):726-741.

2. Eliezer N, Sade J, Silberberg A, Nevo AC. The role of mucus in transport by cilia. Am Rev Respir Dis. 1970;102(1):48-52.

3. Antunes MB, Gudis DA, Cohen NA. Epithelium, cilia, and mucus: their importance in chronic rhinosinusitis. Immunol Allergy Clin North Am. 2009;29(4):631-643.

4. Sanderson MJ, Dirksen ER. Mechanosensitivity of cultured ciliated cells from the mammalian respiratory tract: implications for the regulation of mucociliary transport. Proc Natl Acad Sci U S A. 1986;83(19):7302-7306.

5. Winters SL, Davis CW, Boucher RC. Mechanosen- sitivity of mouse tracheal ciliary beat frequency: roles for $\mathrm{Ca} 2+$, purinergic signaling, tonicity, and viscosity. Am J Physiol Lung Cell Mol Pbysiol. 2007; 292(3):L614-L624.

6. Zhao KQ, et al. Inherent differences in nasal and tracheal ciliary function in response to Pseudomonas aeruginosa challenge. Am J Rhinol Allergy. 2011;25(4):209-213.

7. Wong LB, Miller IF, Yeates DB. Stimulation of tracheal ciliary beat frequency by capsaicin. J Appl Physiol. 1990;68(6):2574-2580.

8. Jain B, Rubinstein I, Robbins RA, Leise KL, Sisson $\mathrm{JH}$. Modulation of airway epithelial cell ciliary beat frequency by nitric oxide. Biochem Biophys Res Commun. 1993;191(1):83-88.

9. Jain B, Rubinstein I, Robbins RA, Sisson JH. TNF- alpha and IL-1 beta upregulate nitric oxide-dependent ciliary motility in bovine airway epithelium. Am J Physiol. 1995;268(6 pt 1):L911-917.

10. Korngreen A, Ma W, Priel Z, Silberberg SD. Extracellular ATP directly gates a cation-selective channel in rabbit airway ciliated epithelial cells. J Physiol. 1998;508(pt 3):703-720.

11. Sanderson MJ, Dirksen ER. Mechanosensitive and beta-adrenergic control of the ciliary beat frequency of mammalian respiratory tract cells in culture. Am Rev Respir Dis. 1989;139(2):432-440.

12. Sutto Z, Conner GE, Salathe M. Regulation of human airway ciliary beat frequency by intracellular pH. J Physiol. 2004;560(pt 2):519-532.

13. Mwimbi XK, Muimo R, Green MW, Mehta A. Making human nasal cilia beat in the cold: a real time assay for 
cell signalling. Cell Signal. 2003;15(4):395-402.

14. Schipor I, Palmer JN, Cohen AS, Cohen NA. Quantification of ciliary beat frequency in sinonasal epithelial cells using differential interference contrast microscopy and high-speed digital video imaging. Am J Rbinol. 2006;20(1):124-127.

15. Salathe M, Bookman RJ. Coupling of [Ca2+]i and ciliary beating in cultured tracheal epithelial cells. J Cell Sci. 1995;108(pt 2):431-440.

16. Korngreen A, Priel Z. Simultaneous measurement of ciliary beating and intracellular calcium. BiophysJ. 1994:67(1):377-380.

17. Lansley AB, Sanderson MJ. Regulation of airway ciliary activity by $\mathrm{Ca} 2+$ : simultaneous measurement of beat frequency and intracellular $\mathrm{Ca} 2+$. Biophys J. 1999;77(1):629-638.

18. Yang B, Schlosser RJ, McCaffrey TV. Signal transduction pathways in modulation of ciliary beat frequency by methacholine. Ann Otol Rhinol Laryngol. 1997;106(3):230-236.

19. Uzlaner N, Priel Z. Interplay between the NO pathway and elevated $[\mathrm{Ca} 2+]$ i enhances ciliary activity in rabbit trachea. J Pbysiol. 1999;516(pt 1):179-190.

20. De Groote MA, Fang FC. NO inhibitions: antimicrobial properties of nitric oxide. Clin Infect Dis. 1995; 21(suppl 2):S162-S165

21. Eberhard A, Burlingame AL, Eberhard C, Kenyon GL, Nealson KH, Oppenheimer NJ. Structural identification of autoinducer of Photobacterium fischeri luciferase. Biochemistry. 1981;20(9):2444-2449.

22. Mitchell RJ, Lee SK, Kim T, Ghim CM. Microbial linguistics: perspectives and applications of microbial cell-to-cell communication. BMB Rep. 2011;44(1):1-10.

23. Braun T, Mack B, Kramer MF. Solitary chemosensory cells in the respiratory and vomeronasal epithelium of the human nose: a pilot study. Rhinology. 2011;49(5):507-512.

24. Shah AS, Ben-Shahar Y, Moninger TO, Kline JN, Welsh MJ. Motile cilia of human airway epithelia are chemosensory. Science. 2009;325(5944):1131-1134.

25. Sbarbati A, et al. Acyl homoserine lactones induce early response in the airway. Anat Rec (Hoboken). 2009;292(3):439-448.

26. Tizzano M, et al. Nasal chemosensory cells use bitter taste signaling to detect irritants and bacterial signals. Proc Natl Acad SciU S A. 2010;107(7):3210-3215.

27. Kim U, Wooding S, Ricci D, Jorde LB, Drayna D. Worldwide haplotype diversity and coding sequence variation at human bitter taste receptor loci. Hum Mutat. 2005;26(3):199-204.

28. Kim UK, Drayna D. Genetics of individual differences in bitter taste perception: lessons from the PTC gene. Clin Genet. 2005;67(4):275-280.

29. Bufe B, et al. The molecular basis of individual differences in phenylthiocarbamide and propylthiouracil bitterness perception. Curr Biol. 2005;15(4):322-327.

30. Tan J, Abrol R, Trzaskowski B, Goddard WA 3rd. The 3D structure prediction of TAS2R38 bitter receptors bound to agonists phenylthiocarbamide (PTC) and 6-n-Propylthiouracil (PROP) [published online ahead of print July 13, 2012]. J Chem Inf Model. doi:10.1021/ci300133a.

31. Biarnes $\mathrm{X}$, et al. Insights into the binding of Phenyltiocarbamide (PTC) agonist to its target human TAS2R38 bitter receptor. PLoS One. 2010;5(8):e12394.

32. Floriano WB, et al. Modeling the human PTC bitter-taste receptor interactions with bitter tastants. JMol Model. 2006;12(6):931-941.

33. Chen MC, Wu SV, Reeve JR Jr, Rozengurt E. Bitter stimuli induce $\mathrm{Ca} 2+$ signaling and CCK release in enteroendocrine STC-1 cells: role of L-type voltagesensitive Ca2+ channels. Am J Physiol Cell Physiol. 2006; 291(4):C726-C739.

34. Nelson TM, Munger SD, Boughter JD. Taste sensitivities to PROP and PTC vary independently in mice. Chem Senses. 2003;28(8):695-704.

35. Wu SV, Chen MC, Rozengurt E. Genomic organiza- tion, expression, and function of bitter taste receptors (T2R) in mouse and rat. Physiol Genomics. 2005; 22(2):139-149.

36. Dimova S, Brewster ME, Noppe M, Jorissen M, Augustijns P. The use of human nasal in vitro cell systems during drug discovery and development. Toxicol In Vitro. 2005;19(1):107-122.

37. Johnson LG, Dickman KG, Moore KL, Mandel LJ, Boucher RC. Enhanced $\mathrm{Na}^{+}$transport in an airliquid interface culture system. Am J Physiol. 1993; 264(6 pt 1):L560-L565.

38. Manders EMM, Verbeek FJ, Aten JA. Measurement of co-localization of objects in dual-colour confocal images. J Microsc. 1993;169(3):375-382.

39. Zhang Y, et al. Coding of sweet, bitter, and umami tastes: different receptor cells sharing similar signalling pathways. Cell. 2003;112(3):293-301.

40. Gulbransen BD, Clapp TR, Finger TE, Kinnamon SC. Nasal solitary chemoreceptor cell responses to bitter and trigeminal stimulants in vitro. J Neurophysiol. 2008;99(6):2929-2937.

41. Kim UK, Jorgenson E, Coon H, Leppert M, Risch N, Drayna D. Positional cloning of the human quantitative trait locus underlying taste sensitivity to phenylthiocarbamide. Science. 2003;299(5610):1221-1225.

42. Gee KR, Brown KA, Chen WN, Bishop-Stewart J, Gray D, Johnson I. Chemical and physiological characterization of fluo- $4 \mathrm{Ca}(2+)$-indicator dyes. Cell Calcium. 2000;27(2):97-106.

43. Yan W, Sunavala G, Rosenzweig S, Dasso M, Brand JG, Spielman AI. Bitter taste transduced by PLCbeta(2)-dependent rise in $\operatorname{IP}(3)$ and alpha-gustducin-dependent fall in cyclic nucleotides. Am J Physiol Cell Physiol. 2001;280(4):C742-C751.

44. Zhang Z, Zhao Z, Margolskee R, Liman E. The transduction channel TRPM5 is gated by intracellular calcium in taste cells. J Neurosci. 2007; 27(21):5777-5786.

45. Charlton TS, et al. A novel and sensitive method for the quantification of $\mathrm{N}$-3-oxoacyl homoserine lactones using gas chromatography-mass spectrometry: application to a model bacterial biofilm. Environ Microbiol. 2000;2(5):530-541.

46. Conway BA, Venu V, Speert DP. Biofilm formation and acyl homoserine lactone production in the Burkholderia cepacia complex. J Bacteriol. 2002; 184(20):5678-5685

47. Chambers CE, Visser MB, Schwab U, Sokol PA. Identification of $\mathrm{N}$-acylhomoserine lactones in mucopurulent respiratory secretions from cystic fibrosis patients. FEMS Microbiol Lett. 2005;244(2):297-304.

48. Frommberger M, et al. Analysis of N-acyl-L-homoserine lactones produced by Burkholderia cepacia with partial filling micellar electrokinetic chromatography-electrospray ionization-ion trap mass spectrometry. Electrophoresis. 2003;24(17):3067-3074.

49. Riedel K, et al. N-acylhomoserine-lactone-mediated communication between Pseudomonas aeruginosa and Burkholderia cepacia in mixed biofilms. Microbiology. 2001;147(pt 12):3249-3262.

50. Malott RJ, Baldwin A, Mahenthiralingam E, Sokol PA. Characterization of the ccilR quorum-sensing system in Burkholderia cenocepacia. Infect Immun. 2005;73(8):4982-4992.

51. Winson MK, et al. Multiple N-acyl-L-homoserine lactone signal molecules regulate production of virulence determinants and secondary metabolites in Pseudomonas aeruginosa. Proc Natl Acad Sci US A. 1995;92(20):9427-9431

52 . Meyerhof $\mathrm{W}$, et al. The molecular receptive ranges of human TAS2R bitter taste receptors. Chem Senses. 2009;35(2):157-170.

53. Ricciardolo FL. Multiple roles of nitric oxide in the airways. Thorax. 2003;58(2):175-182

54. Itoh Y, et al. Determination and bioimaging method for nitric oxide in biological specimens by diaminofluorescein fluorometry. Anal Biochem. 2000; 287(2):203-209.
55. Rees DD, Palmer RM, Schulz R, Hodson HF, Moncada S. Characterization of three inhibitors of endothelial nitric oxide synthase in vitro and in vivo. Br J Pharmacol. 1990;101(3):746-752.

56. Pearson JP, Pesci EC, Iglewski BH. Roles of Pseudomonas aeruginosa las and rhl quorum-sensing systems in control of elastase and rhamnolipid biosynthesis genes. J Bacteriol. 1997;179(18):5756-5767.

57. Antunes MB, Cohen NA. Mucociliary clearance a critical upper airway host defense mechanism and Methods of assessment. Curr Opin Allergy Clin Immunol. 2007;7(1):5-10.

58. Zhang L, Sanderson MJ. The role of cGMP in the regulation of rabbit airway ciliary beat frequency. J Physiol. 2003;551(pt 3):765-776.

59. Zhang L, Sanderson MJ. Oscillations in ciliary beat frequency and intracellular calcium concentration in rabbit tracheal epithelial cells induced by ATP. J Physiol. 2003;546(pt 3):733-749.

60. O'Toole GA, Kolter R. Flagellar and twitching motility are necessary for Pseudomonas aeruginosa biofilm development. Mol Microbiol. 1998;30(2):295-304.

61. Lundberg JO. Nitric oxide and the paranasal sinuses. Anat Rec (Hoboken). 2008;291(11):1479-1484.

62. Roudnitzky N, et al. Genomic, genetic and functional dissection of bitter taste responses to artificial sweeteners. Hum Mol Genet. 2011;20(17):3437-3449.

63. Behrens M, Meyerhof W. Mammalian bitter taste perception. Results Probl Cell Differ. 2009;47:203-220.

64. Temussi P. The sweet taste receptor: a single receptor with multiple sites and modes of interaction. Adv Food Nutr Res. 2007;53:199-239.

65. Schwarzer C, et al. Pseudomonas aeruginosa biofilm-associated homoserine lactone $\mathrm{C} 12$ rapidly activates apoptosis in airway epithelia. Cell Microbiol. 2012;14(5):698-709.

66. Pearson JP, Passador L, Iglewski BH, Greenberg EP. A second $\mathrm{N}$-acylhomoserine lactone signal produced by Pseudomonas aeruginosa. Proc Natl Acad SciUS A. 1995;92(5):1490-1494.

67. Pearson JP, et al. Structure of the autoinducer required for expression of Pseudomonas aeruginosa virulence genes. Proc Natl Acad Sci U S A. 1994; 91(1):197-201.

68. Pearson JP, Van Delden C, Iglewski BH. Active efflux and diffusion are involved in transport of Pseudomonas aeruginosa cell-to-cell signals. J Bacteriol. 1999;181(4):1203-1210.

69. Wooding S, Kim UK, Bamshad MJ, Larsen J, Jorde LB, Drayna D. Natural selection and molecular evolution in PTC, a bitter-taste receptor gene. Am J Hum Genet. 2004;74(4):637-646.

70. Dong D, Jones G, Zhang S. Dynamic evolution of bitter taste receptor genes in vertebrates. BMC Evol Biol. 2009;9:12.

71. Hancock AM, et al. Adaptations to climate-mediated selective pressures in humans. PLoS Genet. 2011; 7(4):e1001375.

72. Hekiert AM, et al. Biofilms correlate with TH1 inflammation in the sinonasal tissue of patients with chronic rhinosinusitis. Otolaryngol Head Neck Surg. 2009;141(4):448-453.

73. Lai Y, Chen B, Shi J, Palmer JN, Kennedy DW, Cohen NA. Inflammation-mediated upregulation of centrosomal protein 110, a negative modulator of ciliogenesis, in patients with chronic rhinosinusitis. J Allergy Clin Immunol. 2011;128(6):1207-1215.

74. Ramanathan M Jr, Lane AP. A comparison of experimental Methods in molecular chronic rhinosinusitis research. Am J Rhinol. 2007;21(3):373-377.

75. Savitski AN, Mesaros C, Blair IA, Cohen NA, Kreindler JL. Secondhand smoke inhibits both Cl-and $\mathrm{K}+$ conductances in normal human bronchial epithelial cells. Respir Res. 2009;10:120.

76. Zhao KQ, Xiong G, Wilber M, Cohen NA, Kreindler $\mathrm{JL}$. A role for two-pore $\mathrm{K}+$ channels in modulating $\mathrm{Na}+$ absorption and $\mathrm{Cl}$ - secretion in normal human bronchial epithelial cells. Am J Physiol Lung 
Cell Mol Physiol. 2012;302(1):L4-L12.

77. Reszka KJ, O’Malley Y, McCormick ML, Denning GM, Britigan BE. Oxidation of pyocyanin, a cytotoxic product from Pseudomonas aeruginosa, by microperoxidase 11 and hydrogen peroxide. Free Radic Biol Med. 2004;36(11):1448-1459.

78. de Prost N, Saumon G. Glucose transport in the lung and its role in liquid movement. Respir Physiol Neurobiol. 2007;159(3):331-337.

79. Pezzulo AA, et al. Glucose depletion in the airway surface liquid is essential for sterility of the airways. PLoS One. 2011;6(1):e16166.

80. Baker EH, Wood DM, Brennan AL, Clark N, Baines DL, Philips BJ. Hyperglycaemia and pulmonary infection. Proc Nutr Soc. 2006;65(3):227-235.

81. Baker EH, et al. Hyperglycemia and cystic fibrosis alter respiratory fluid glucose concentrations estimated by breath condensate analysis. J Appl Physiol. 2007;102(5):1969-1975.

82. Sisson JH, Stoner JA, Ammons BA, Wyatt TA. All-digital image capture and whole-field analysis of ciliary beat frequency. J Microsc. 2003;211(pt 2):103-111.

83. Edelstein A, Amodaj N, Hoover K, Vale R, Stuurman $\mathrm{N}$. Computer control of microscopes using microManager. Curr Protoc Mol Biol Chapter. 2010;Chapter 14 :Unit 14.20.

84. Ireson NJ, Tait JS, MacGregor GA, Baker EH. Comparison of nasal $\mathrm{pH}$ values in black and white individuals with normal and high blood pressure. Clin Sci (Lond). 2001;100(3):327-333.

85. Yoon SS, et al. Anaerobic killing of mucoid Pseudomonas aeruginosa by acidified nitrite derivatives under cystic fibrosis airway conditions. J Clin Invest. 2006;116(2):436-446.

86. Butler AR, Feelisch M. Therapeutic uses of inorganic nitrite and nitrate: from the past to the future. Circulation. 2008;117(16):2151-2159.

87. Fischer $\mathrm{H}$, Widdicombe JH. Mechanisms of acid and base secretion by the airway epithelium. J Membr Biol. 2006;211(3):139-150.

88. Steinke JW, Woodard CR, Borish L. Role of hypoxia in inflammatory upper airway disease. Curr Opin
Allergy Clin Immunol. 2008;8(1):16-20.

89. Matsune S, Kono M, Sun D, Ushikai M, Kurono Y. Hypoxia in paranasal sinuses of patients with chronic sinusitis with or without the complication of nasal allergy. Acta Otolaryngol. 2003;123(4):519-523.

90. Bals R, Wang X, Zasloff M, Wilson JM. The peptide antibiotic LL-37/hCAP-18 is expressed in epithelia of the human lung where it has broad antimicrobial activity at the airway surface. Proc Natl Acad Sci US A. 1998;95(16):9541-9546.

91. Singh PK, et al. Production of beta-defensins by human airway epithelia. Proc Natl Acad Sci U S A. 1998;95(25):14961-14966.

92. Singh PK, Tack BF, McCray PB Jr, Welsh MJ. Synergistic and additive killing by antimicrobial factors found in human airway surface liquid. Am J Physiol Lung Cell Mol Physiol. 2000;279(5):L799-L805.

93. Reed DR, et al. The perception of quinine taste intensity is associated with common genetic variants in a bitter receptor cluster on chromosome 12 . Hum Mol Genet. 2010;19(21):4278-4285. 